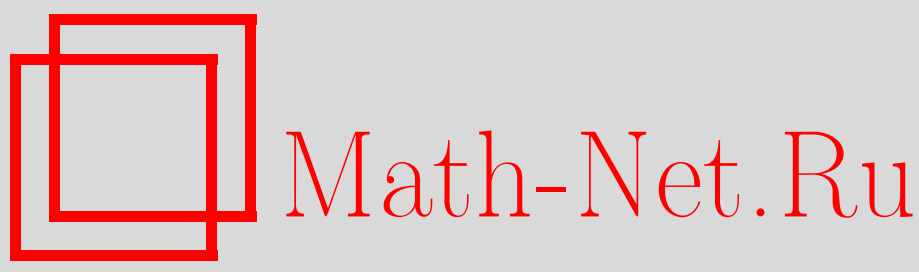

О. В. Мельников, Асферические про-р-группы, Матем. сб., 2002, том 193, номер 11, 71-104

DOI: https://doi.org/10.4213/sm692

Использование Общероссийского математического портала Math-Net.Ru подразумевает, что вы прочитали и согласны с пользовательским соглашением

http://www . mathnet.ru/rus/agreement

Параметры загрузки:

IP : 44.207 .124 .84

26 апреля 2023 г., 05:00:00 


\title{
О.В. Мельников
}

\section{Асферические про- $p$-группы}

\begin{abstract}
В статье вводится понятие асферической про- $p$-группы. Доказано, что если группа $G=F / N$ асферическая, где $F$ - свободная про-р-группа, то для $\mathbb{F}_{p}[[G]]$-модуля соотношений $\bar{N}=N / N^{p}[N, N]$ справедливо утверждение типа теоремы Линдона о тождествах. Описаны конечные подгруппы и центр $G$. Исследована структура асферической про-p-групшы $G$, имеюшей разрешимую нормальную подгруппу $A \neq\{1\}$. В частности, если $A \cong \mathbb{Z}_{p}$, то $G$ содержит подгруппу конечного индекса вида $A \lambda W$, где $W$ - свободная про- $p$-группа.

Библиография: 25 названий.
\end{abstract}

\section{Введение}

Настоящая статья представляет собой фрагмент комбинаторной теории про- $p$ групп, связанный с их заданием порождаюшими и определяющими соотношениями. Объектом исследования являются про- $p$-групшы, между определяюшими соотношениями которых не существует нетривиальных зависимостей. Содержание работы (с учетом того, что предполагается подготовить продолжение настоящей статьи) примерно соответствует результатам о дискретных группах, составляющим гл. III книги [1], и их развитию в [2] и [3]. Специфика про-р-групп проявляется в том, что нам достаточно работать на уровне цепных комплексов над полем $\mathbb{F} p$, соответствуюших двумерным комплексам Кэли.

Пусть $G=F / N$, где $F$ - некоторая свободная про-р-группа. Модулем соотношений группь $G$ для этого копредставления назовем $\mathbb{F}_{p}(G)$-модуль $\bar{N}=N / N^{*}$, где $N^{*}=N^{p}[N, N]$. В качестве определения асферичности про-pгрупп удобно принять следующую цепную интерпретацию асферичности их комплексов Кэли.

ОПРЕДЕЛЕНИЕ. Про- $p$-группа $G$ называется асферической, если какой-либо из ее модулей соотношений является пермутационньм $\mathbb{F}_{p}(G)$-модулем $\mathbb{F}_{p}\left(T, t^{0}\right)$, определяемым проконечным (иначе, булевьм) пунктированным $G$-пространством $\left(T, t^{0}\right)$.

Определение пермутационного модуля $\mathbb{F}_{p}\left(T, t^{0}\right)$ см. в (1.8). Одна из причин, по которой здесь, вопреки традиции, модулем соотношений названа не факторгруппа $N /[N, N]$, а именно $N / N^{*}$, состоит в том, что это априори позволяет охватить определением более широкий класс про-р-групп.

Представим полученные в статье результаты, разбив их на несколько блоков. В первый блок соберем те из них, которые формально касаются определения класса асферических про- $p$-групп.

Прежде всего следует отметить, что (в отличие от теории дискретњых групп) асфферичность является в действительности свойством самой про-p-группы $G$, а

(C) О.в. Мельников 2002 
не ее конкретного копредставления. Точнее, пермутационность одного из модулей соотношений группы $G$ влечет пермутационность модулей соотношений, соответствующих всем ее копредставлениям. (Это вытекает ввиду леммы (2.3) из теоремы (2.5), лежащей в основе построенной здесь теории асферических про-р-групп.) Более того, базисные $G$-пространства всех модулей соотношений данной асферической про- $p$-группы $G$ по существу однозначно определяются в терминах группы $G$ (см. теорему (3.5)).

Непосредственно из приведенного вьше определения вытекает, что подгруппы асферических групп также асферичны. Очевидные примеры асферических групп дают конечные циклические $p$-группы (и по теореме (2.7) других конечных асферических $p$-групп нет), а также свободные про-p-группы. Асферичность сохраняется при образовании ряда свободных конструкций в классе про-p-групп (см. теорему (2.8) и следствия из нее).

Далее, среди асферических групп целиком содержится класс про- $p$-групп $G$ когомологической размерности $\mathrm{cd} G \leqslant 2$ (так как модули соотношений таких $G$ свободны и, следовательно, пермутационны (см., например, [4])). Следующая теорема показывает, что если бы в определении асферичности использовались обычные, не пунктированные $G$-пространства, то полученный класс групп оказался бы не намного шире.

Теорема А. Асферическая про-р-группа $G$ обладает модулем соотношений, являюшимся пермутационным над некоторым не пунктированным G-пространством, тогда и только тогда, когда она содержит подгруппу $H$ конечного индекса $c$ cd $H \leqslant 2$.

Этот результат выводится в (3.8) из более точного утверждения теоремы (3.7). Отметим здесь же следствие (3.6), согласно которому каждая асферическая про-pгруппа без кручения имеет когомологическую размерность не более 2.

Второй блок составляют результаты о специальных свойствах $G$-пространств $\left(T, t^{0}\right)$, определяюших модули соотношений $N / N^{*}$ асферических про-p-групш $G=$ $F / N$, и о расположении этих пространств в факторгруппах $F / N^{*}$. Они представлены в теоремах (3.1) и (3.2) (см. также часть (а) теоремы (3.5)). Должным образом интерпретированные эти теоремы дают утверждения об асферических про-pгруппах, дискретными прототипами которых являются хорошо известные результаты о группах с одним определяющим соотношением. В частности, теорему (3.2) следует рассматривать как адекватную про- $p$-ситуации формулировку утверждения типа теоремы Линдона о тождествах (см. [1]).

Приведем здесь содержащиеся в теоремах (3.1) и (3.2) результаты в традиционной форме. Условимся называть максимальным корнем из әлемента $r$ свободной $n$ ро -p -групnь $F$ такой элемент $h$, порождающий централизатор $C_{F}(r) \cong \mathbb{Z}_{p}$, что $r=h^{q}$ для $q=p^{m} \geqslant 1$. Число $q$ естественно называть степенью корня $h$.

ТеОРема В. Пусть $G=F / N$ - асферическая про-р-группа, где $F-$ свободная про-р-группа. Тогда для некоторого проконечного пространства $X=I \cup$ $\{*\}$ существуют такие элементы $r_{i} \in N, i \in I$, порождающие $N$ как нормальную подгруппу $F$, что

(а) $\bar{N}=\bigoplus_{x \in X} A_{x}-$ прямая сумма над $X$ в смысле статьи [5] ииклических подмодулей $A_{i}$, порохсденных образами $r_{i}$ в $\bar{N}, i \in I$, и нулевого $A_{*}=\{0\}$ 
(b) для $i \in I$ модуль $A_{i}$ канонически изоморфен фактормодулю $\mathbb{F}_{p}(G) / \mathbb{F}_{p}(G)\left(s_{i}-1\right)$, әде $s_{i}-$ образ в $G$ максимального корня из $r_{i} \in F\left(\right.$ при этом порядок $s_{i}$ совпадает со степенью корня);

(c) если $K$ - нетривиальная конечная подгруппа $G$, то существуют единственные индекс $i \in I$ и смежсный класс $g S_{i}$ по ииклической подгруппе $S_{i}=\left\langle s_{i}\right\rangle$, для которых $K \subseteq g S_{i} g^{-1}$.

Пространство $X$ в этой теореме есть не что иное, как пространство $G$-орбит $G \backslash T$ для $G$-пространства $\left(T, t^{0}\right)$ из определения асфферической группы (см. (3.3)). Отметим, что в такой формулировке теряется информация о том, что $\bar{N}$ - пермутационньй модуль.

Третий блок результатов посвяшен детальному изучению структуры асферических про- $p$-групп с нетривиальньми разрешимыми нормальньми подгруппами. Эти результаты, составляющие $\S 4$, можно понимать также как описание строения нормализаторов (и централизаторов) абелевых подгруп в асферических про-pгруппах. В концентрированном виде полученная в $\S 4$ информация содержится в описании разрешимых асферических про-р-групп и в следуюшей теореме (см. теоремы (4.9) и (4.5)).

Теорема С. Произвольная асферическая про-р-группа $G$ обладает наибольшей разрешимой нормальной подгруппой $R(G)$. Если $G$ - неразрешимая асферическая про-р-группа с нетривиальным радикалом $R(G)$, то

(а) $R(G) \cong \mathbb{Z}_{p}$ и в группе $G$ содержится подгруппа без кручения индекса не более 2 (, следовательно, $G$ - группа без кручения при $p \neq 2$ );

(b) если $G$ без кручения, то $d(G)=r(G)+1$, где $d(G)$ и $r(G)$ - минимальные числа пороэсдающих и соответственно определяющих соотношений гpynnвь $G$;

(c) $G$ обладает подгруппой конечного индекса, имеющей вид $R(G) \lambda W$, где $W$ - свободная про-р-группа ранга не менее 2.

Утверждения (a) и (b) этой теоремы напоминают теорему Мурасуги о центре для дискретных одно-определенных групп (с той разницей, что в теореме С кардинальные числа $d(G)$ и $r(G)$ могут быть бесконечны). Соответствующий результат о неабелевых асфферических про- $p$-группах с нетривиальньм центром содержится в теореме (4.6).

Класс абелевых асферических про-р-групп описать легко: это группы, изоморфные $\mathbb{Z} / p^{m} \mathbb{Z}, \mathbb{Z}_{p}$ или $\mathbb{Z}_{p} \times \mathbb{Z}_{p}$. Что касается разрешимых асферических про-ргрупп, то в $\S 5$ мы сформулируем теорему, дающую их полное описание. Доказательство этой теоремы планируется опубликовать позднее. В настоящей работе мы ограничимся доказательством следующего результата.

Теорема D. Если $p \geqslant 5$, то неабелева разрешимая асферическая про-р группа изоморфна полупрямому произведению $\mathbb{Z}_{p} 入 \mathbb{Z}_{p}$.

(Так как $\operatorname{cd}\left(\mathbb{Z}_{p} \lambda \mathbb{Z}_{p}\right)=2$, то эта группа асфферична.)

Первый параграф̆ статьи содержит некоторым образом систематизированное изложение утверждений о проконечных модулях, которые, как правило, являются леммами для доказательств результатов $\S \S 2,3$. Хотелось бы, однако, выделить одно утверждение, представляющее самостоятельный интерес. Это предло- 
жение (1.16) о существовании и единственности так называемых сердцевин в проконечных модулях над про-р-группами.

Именно это предложение позволяет получить уже упоминавшуюся базисную теорему (2.5). Мы все же приведем здесь утверждение этой теоремы в следующей, слегка усеченной форме.

Теорема Е. Асферичность про-р-группь $G$ әквивалентна тому, что в некоторой (а тогда и в каждой) точной последовательности проконечных $\mathbb{F}_{p}(G)$-модулей

$$
0 \rightarrow M \rightarrow P_{1} \rightarrow P_{0} \rightarrow \mathbb{Z} / p \mathbb{Z} \rightarrow 0
$$

со свободными модулями $P_{0}$ и $P_{1}$ модуль $M$ является пермутационным.

Это утверждение позволяет несколько иначе взглянуть на понятие асферичности про- $p$-групп. Дело в том, что наиболее естественный путь возникновения последовательностей вида $(*)$ состоит в построении цепных комплексов над $\mathbb{F}_{p}$ по двумерньп про- $p$-комплексам, являющимся связными, односвязными и асферическими, на которых про-р-группа $G$ действует с тривиальными стабилизаторами вершиин.

Таким образом, изучение асфферических про-р-групп можно рассматривать как распространение в размерность 2 исследований проконечных групп, действующих на проконечных деревьях, начатых в [6] и развитых в [7] и [8] до построения проконечного варианта теории Басса-Серра.

\section{§1. Свободные и пермутационные модули}

(1.1) Через $\mathbb{F}_{p}(G)$ будем обозначать пополненное групповое кольцо про- $p$ группы $G$ над полем $\mathbb{F}_{p}$ из $p$ элементов. Его определение содержится, например, в $[9]$ и $[4]$, где используется более традиционное обозначение $\mathbb{F}_{p}[[G]]$.

В этой статье мы ограничимся рассмотрением проконечных $\mathbb{F}_{p}(G)$-модулей, считая их, как правило, левыми. Для краткости будем называть их просто $G$-модyлями. Все они являются, очевидно, абелевыми про-p-группами экспоненты $p$ и, следовательно, изоморфны прямым произведениям $\prod_{i \in I} \mathbb{Z} / p \mathbb{Z}$.

Так как $\mathbb{Z} / p \mathbb{Z}$ - единственный простой $G$-модуль, то кольцо $\mathbb{F}_{p}(G)$ является локальньм (топологически) кольцом с радикалом Джекобсона $I(G)$. Здесь и везде ниже $I(G)$ означает фундаментальный идеал $\mathbb{F}_{p}(G)$, являющийся по определению ядром гомоморфизма аугментации $\varepsilon: \mathbb{F}_{p}(G) \rightarrow \mathbb{F}_{p}$, который отображает в единицу все элементы из $G \subset \mathbb{F}_{p}(G)$.

ЗАмЕчАниЕ. Практически все приведенные ниже результаты справедливы в более общей ситуации: по существу, важна лишш локальность кольца и полнота модулей. В ряде доказательств нужно будет только заменить $I(G)$ на радикал Джекобсона.

(1.2) Свободный $G$-модуль ранга $\mathfrak{m} \geqslant 0$ определим как прямое произведение

$$
\mathbb{F}_{p}(G)^{\mathfrak{m}}=\prod_{i \in I} A_{i}, \quad A_{i}=\mathbb{F}_{p}(G)
$$

$\mathfrak{m}=|I|$ экземпляров свободного модуля ранга 1. (Основанием для такого определения является лемма (1.5).) Роль свободного базиса этого модуля играет подмножество $X=\left\{x_{i} \mid i \in I\right\}$, где $x_{i}$ - единица кольца $\mathbb{F}_{p}(G)=A_{i}$. 
Иногда нам будет удобно элемент $\eta=\left(\eta_{i}\right), \eta_{i} \in \mathbb{F}_{p}(G)$, из $\mathbb{F}_{p}(G)^{\mathfrak{m}}$ записывать, используя базис $X$, в виде $\eta=\sum_{i \in I} \eta_{i} x_{i}$. Корректность этой записи следует из того, что $X$ является подмножеством, сходящимся к нулю (т.е. произвольная окрестность нуля содержит почти все элементы из $X)$.

(1.3) Для $G$-модуля $M$ через $M^{G}$ обозначим множество элементов из $M$, неподвижных при действии $G$.

Лемма. Если $P$ - свободный $G$-модуль и группа $G$ бесконечна, то $P^{G}=\{0\}$.

ДокаЗАТЕЛьство. Ясно, что достаточно рассмотреть случай $P=\mathbb{F}_{p}(G)$. Необходимое равенство $\mathbb{F}_{p}(G)^{G}=\{0\}$ является частным случаем более общего утверждения из [10]. Для полноты изложения приведем здесь схему доказательства в этом частном случае.

Пусть $G=\lim G / U_{\lambda}$, где $G / U_{\lambda}$ - конечные $p$-группы. Тогда, очевидно, $\mathbb{F}_{p}(G)^{G}=\lim _{\mathbb{F}} \mathbb{F}_{p}\left(G / U_{\lambda}\right)^{G / U_{\lambda}}$. Легко проверить, что $\mathbb{F}_{p}\left(G / U_{\lambda}\right)^{G / U_{\lambda}}$ состоит из элементов вида $a \nu_{\lambda}$, где $a \in \mathbb{F}_{p}, \nu_{\lambda}$ - сумма всех элементов из $G / U_{\lambda}$. Поэтому для $\mu>\lambda$ ограничение на $\mathbb{F}_{p}\left(G / U_{\mu}\right)^{G / U_{\mu}}$ проекции $\mathbb{F}_{p}\left(G / U_{\mu}\right) \rightarrow \mathbb{F}_{p}\left(G / U_{\lambda}\right)$ проективного предела является умножением на $\left[U_{\lambda}: U_{\mu}\right]=p^{\alpha}, \alpha>0$, т.е. нулевьм гомоморфизмом.

(1.4) Условимся через $A \otimes_{G} M$ обозначать введенное в [9] пополненное тензорное произведение над кольцом $\mathbb{F}_{p}(G)$ правого $G$-модуля $A$ и левого $G$-модуля $M$.

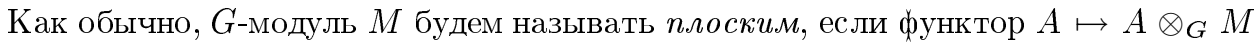
является точным.

Лемма. Для про-р-группь $G$ следующие свойства $G$-модуля $P$ эквивалентны:

(i) $P-$ свободныц модуль;

(ii) $P$ - проективныцй модуль;

(iii) $P-$ плоский модуль.

ДокАЗАТЕЛьство. Импликация (ii) $\Longrightarrow$ (i) устанавливается стандартньми рассуждениями, использующими локальность $\mathbb{F}_{p}(G)$. Отметим, что в качестве свободного базиса $P$ может быть взято произвольное сходящееся к нулю подмножество $\left\{x_{i} \mid i \in I\right\}$ со следующим свойством: $P / I(G) P=\prod_{i \in I} C_{i}$, где подгрупа $C_{i} \cong \mathbb{Z} / p \mathbb{Z}$ порождена образом элемента $x_{i}, i \in I$.

Справедливость импликаций (iii) $\Longrightarrow$ (ii) и (i) $\Longrightarrow$ (iii) установлена в $[9$; предложение 3.1, лемма 2.1].

(1.5) В настояшей статье сушественным образом будет использоваться понятие прямой суммы (копроизведения) семейства модулей, непрерывно индексированных точками топологического пространства, введенное в [5]. Приведем здесь соответствующие определения в удобной для использования форме.

Пусть $X$ - проконечное (иначе, булево) пространство. Семейство $\Delta=\left\{A_{x} \mid\right.$ $x \in X\}$ подмодулей $G$-модуля $A$ считается непрерывно индексированным точками $X$, если для любой окрестности нуля $W$ в $A$ подмножество $\left\{x \in X \mid A_{x} \subseteq W\right\}$ открыто в $X$.

Модуль $A$ является по определению прямой суммой $\bigoplus_{x \in X} A_{x}$ такого семейства $\Delta$, если

(1) подмодули $A_{x}, x \in X$, порождают $A$; 
(2) $A_{x} \cap A_{y}=\{0\}$ при $x \neq y$;

(3) для любого $G$-модуля $B$ произвольный набор гомоморфизмов $\psi_{x}: A_{x} \rightarrow B$, $x \in X$, который определяет непрерывное отображение $\psi$ подпространства $\bigcup_{x \in X} A_{x} \subset A$ в $B$, продолжается до гомоморфизма $\varphi: A \rightarrow B$ (т.е. $\left.\varphi\right|_{A_{x}}=$ $\psi_{x}$ для всех $\left.x \in X\right)$.

Представление модуля $B$ в виде проективного предела его конечных фактормодулей позволяет при проверке условия (3) ограничиться рассмотрением только конечных $B$.

ЛЕмма. Прямое произведение $\prod_{i \in I} A_{i}$ множсества $G$-модулей является прямой суммой $\bigoplus_{i \in I \cup\{\infty\}} A_{i}$ над одноточечной компактификацией $I \cup\{\infty\}$ дискретного пространства $I$, әде $A_{\infty}=\{0\}$.

ДоКАЗАТЕЛЬСтво. Из определения топологии прямого произведения вытекает, во-первых, что семейство подмодулей $\Delta$, состоящее из $A_{\infty}=\{0\}$ и модулей $A_{i}$, $i \in I$, естественньм образом вложенных в $\prod_{i \in I} A_{i}$, непрерывно индексировано.

Во-вторых, если $\psi: \bigcup_{i \in I} A_{i} \rightarrow B$-непрерьвное отображение в конечный $G$-модуль $B$ со свойством $\psi(0)=\psi_{\infty}\left(A_{\infty}\right)=\{0\}$, то гомоморфизмы $\psi_{i}=\left.\psi\right|_{A_{i}}$ являются нулевыми для всех $i \in I$, не принадлежащих некоторому конечному подмножеству $J \subseteq I$. Поэтому в качестве гомоморфизма $\varphi$ можно взять композит проекции на $\prod_{j \in J} A_{j}=\bigoplus_{j \in J} A_{j}$ и гомоморфизма $\bigoplus_{j \in J} \psi_{j}$.

(1.6) Лемма. (а) Пусть $\left\{P_{\lambda} \mid \lambda \in \Lambda\right\}$ - произвольная проективная система свободных $G$-модулей. Тогда предел $P=\lim P_{\lambda}$ такәсе является свободным G-модулем.

(b) Прямая сумма $P=\bigoplus_{x \in X} P_{x}$ непрерьвного семейства свободных $G$-модулей также является свободным $G$-модулем.

ДокАЗАТЕЛЬСтво. В силу леммы (1.4) достаточно доказать, что модуль $P$ является проективным.

(a) Рассуждения, аналогичные проведенньм в доказательстве предложения 1 из [11], показывают, что существование поднятия произвольного гомоморфизма $\varphi: P \rightarrow A / K$ до гомоморфизма $\psi: P \rightarrow A$ достаточно установить только для конечных модулей $A$. В этом случае найдется такой индекс $\lambda \in \Lambda$, что $\varphi=\varphi_{\lambda} \rho_{\lambda}$ для некоторого гомоморфизма $\varphi_{\lambda}: P_{\lambda} \rightarrow A / K$, где $\rho_{\lambda}-$ проекция $P \rightarrow P_{\lambda}$. В силу проективности $P_{\lambda}$ сушествует поднятие $\psi_{\lambda}: P_{\lambda} \rightarrow A$ гомоморфизма $\varphi_{\lambda}$. Тогда $\psi=\psi_{\lambda} \rho_{\lambda}-$ искомое поднятие $\varphi$.

(b) Согласно лемме (3.3) из [5] для любого $q \geqslant 0$ и произвольного правого $G$-модуля $A$

$$
\operatorname{Tor}_{q}^{G}(A, P) \cong \bigoplus_{x \in X} \operatorname{Tor}_{q}^{G}\left(A, P_{x}\right)
$$

где $\operatorname{Tor}_{q}^{G}$ - производные функторы пополненного тензорного произведения (см. [9]). Проективность $P_{x}$ в силу леммы (1.4) эквивалентна равенству $\operatorname{Tor}_{1}^{G}\left(A, P_{x}\right)=\{0\}$. Поэтому $\operatorname{Tor}_{1}^{G}(A, P)=\{0\}$, откуда вытекает проективность модуля $P$.

(1.7) По аналогии с [12] введем понятие $\mathbb{F}_{p}$-модуля, свободного над пунктированным проконечным пространством $\left(T, t^{0}\right)$. 
Через $\mathbb{F}_{p}\left(T, t^{0}\right)$ будем обозначать такой проконечный $\mathbb{F}_{p}$-модуль, что сушествует вложение $\omega: T \rightarrow \mathbb{F}_{p}\left(T, t^{0}\right)$, отображающее отмеченную точку $t^{0}$ в нуль и обладающее следующим универсальньп свойством:

$(*)$ для любого непрерывного отображения $\beta: T \rightarrow B, \beta\left(t^{0}\right)=0$, где $B$ - проконечный $\mathbb{F}_{p}$-модуль, сушествует единственный гомоморфизм модулей $\varphi: \mathbb{F}_{p}\left(T, t^{0}\right) \rightarrow B$, удовлетворяющий равенству $\varphi \omega=\beta$.

Так же, как в (1.5), замечаем, что свойство $(*)$ достаточно проверить лишш для конечных модулей $B$. Из этого факта немедленно следует, что если $\left(T, t^{0}\right)=$ $\lim _{\longleftarrow}\left(T_{\lambda}, t_{\lambda}^{0}\right)$, то $\mathbb{F}_{p}\left(T, t^{0}\right)=\lim _{\longleftarrow} \mathbb{F}_{p}\left(T_{\lambda}, t_{\lambda}^{0}\right)$.

Так как для конечного пунктированного пространства $\left(T_{\lambda}, t_{\lambda}^{0}\right)$ в качестве $\mathbb{F}_{p}\left(T_{\lambda}, t_{\lambda}^{0}\right)$ можно взять свободный $\mathbb{F}_{p}$-модуль с базисом $T_{\lambda}-\left\{t_{\lambda}^{0}\right\}$, то мы приходим к явной конструкции модуля $\mathbb{F}_{p}\left(T, t^{0}\right)$.

Из этого явного представления вытекает, в частности, что для бесконечного пространства $T$

$$
\mathbb{F}_{p}\left(T, t^{0}\right) \cong \prod_{i \in I} \mathbb{Z} / p \mathbb{Z}
$$

где мощность множества $I$ равна весу $w(T)$ топологического пространства $T$. (В самом деле, $w(T)$ равен, как легко видеть, мощности множества индексов $\lambda$ в представлении $\left(T, t^{0}\right)=\lim \left(T_{\lambda}, t_{\lambda}^{0}\right)$, где $T_{\lambda}$ - конечные факторпространства $T$.)

Совершенно аналогично определяется свободньй $\mathbb{F}_{p}$-модуль $\mathbb{F}_{p}(S)$ над не пунктированным проконечным пространством $S$. Чтобы не рассматривать отдельно такие модули, удобно отождествлять $\mathbb{F}_{p}(S)=\mathbb{F}_{p}\left(T, t^{0}\right)$, где $T=S \cup\left\{t^{0}\right\}$ и отмеченная точка $t^{0}$ изолирована в $T$.

Небесполезно отметить, что $\mathbb{F}_{p}\left(\left\{t^{0}\right\}, t^{0}\right)=\{0\}$.

(1.8) Пусть $G$-про- $p$-группа. Пунктированное проконечное пространство $\left(T, t^{0}\right)$ называется $G$-пространством, если $G$ непрерывно действует на $T$ гомеоморфизмами, оставляющими на месте отмеченную точку $t^{0}$.

ОПРЕДЕЛЕНИЕ. Пермутаиионнылм $G$-модулем называется модуль $\mathbb{F}_{p}\left(T, t^{0}\right)$, где $\left(T, t^{0}\right)$ - какое-либо $G$-пространство, а действие элемента $g \in G$ определяется как автоморфизм этого модуля, продолжающий (по универсальному свойству (*) из (1.7)) отображение $t \mapsto g \cdot t$ из $T$ в $T \subset \mathbb{F}_{p}\left(T, t^{0}\right)$.

В дальнейшем будут важны также пермутационные $G$-модули $\mathbb{F}_{p}(S)$ над $G$-пространствами $S$, не являющимися пунктированными. В частности, пермутационньми являются модули $\mathbb{F}_{p}(G / H)$, действие $G$ на которых определяется левьм регулярным действием $G$ на пространстве $G / H$ смежных классов по подгрупше $H$.

(1.9) Таким образом, свободный $G$-модуль $\mathbb{F}_{p}(G)$ ранга 1 является пермутационньм. Пермутационность произвольного свободного $G$-модуля вытекает из следующей леммы.

Лемма. Пусть $M_{i}, i \in I,-$ пермутационные $G$-модули. Тогда прямое произведение $M=\prod_{i \in I} M_{i}$ также является пермутационным.

ДоКАЗАТЕЛЬСтво. Пусть $M_{i}=\mathbb{F}_{p}\left(T_{i}, t_{i}^{0}\right)$. Будем считать, что $G$-пространства $T_{i}$ содержатся в модулях $M_{i}$, естественньп образом вложенных в $G$-модуль $M$. При этом $t_{i}^{0}$ совпадает с нулем модуля $M$ для всех $i \in I$. 
Через $T$ обозначим $G$-подпространство в $M$, равное объединению всех $T_{i}, i \in I$, с отмеченной точкой $t^{0}=0$. Из определения тихоновской топологии вытекает замкнутость $T$. С помощью леммы $(1.5)$, в которой полагаем $G=\{1\}$, легко показать, что $M=\mathbb{F}_{p}\left(T, t^{0}\right)$.

(1.10) В дальнейшем мы, как правило, будем считать пунктированное пространство $\left(T, t^{0}\right)$ подпространством в $\mathbb{F}_{p}\left(T, t^{0}\right)$; при этом $t^{0}=0$. Для замкнутого подпространства $S \subseteq T$ через $\langle S\rangle$ будем обозначать порожденный им $\mathbb{F}_{p}$-подмодуль.

ЛЕмма. Для $G$-пространства $\left(T, t^{0}\right)$ справедливь следуюшие утвержсдения.

(а) Пусть $\delta: T \rightarrow X-$ такое непрерьвное отображсние на некоторое проконечное пространство $X$, что $\delta(g \cdot t)=\delta(t)$ для всех $g \in G$, $t \in T$. Тогда пермутационныи $G$-модуль $\mathbb{F}_{p}\left(T, t^{0}\right)=\bigoplus_{x \in X} A_{x}$, где $A_{x}=\left\langle\delta^{-1}(x)\right\rangle$.

(b) Пусть $S-G$-инвариантное подпространство T. Тогда $\langle S\rangle$ естественным образом изоморфен пермутационному $G$-модулю $\mathbb{F}_{p}\left(S, t^{0}\right)$, если $t^{0} \in S$, или $\mathbb{F}_{p}(S)$, если $t^{0} \notin S$.

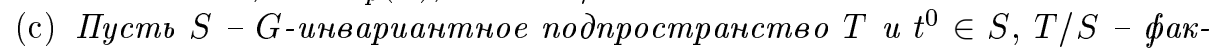
торпространство $T$, полученное стягиванием $S$ в точку $s^{0}$. Тогда $\left(T / S, s^{0}\right)$ - пунктированное G-пространство и пермутационный $G$-модуль $\mathbb{F}_{p}\left(T / S, s^{0}\right)$ естественным образом изоморфен фактормодулю $\mathbb{F}_{p}\left(T, t^{0}\right) /\langle S\rangle$.

ДоКАЗАТЕЛЬСТВА всех утверждений представляют собой, по существу, полутривиальную проверку универсальных свойств из определений (1.5) и (1.7). За деталями можно обратиться в [5], где подобная проверка проделана для более сложного случая копроизведений в категории проконечных групп.

(1.11) Пусть $T$ - некоторое $G$-пространство. Через $G_{t}$ условимся обозначать стабилизатор в $G$ точки $t \in T$, а через $G \backslash T$-пространство $G$-орбит в $T$, снабженное фактортопологией.

Для удобства ссылок сформулируем следствие из предыдущей леммы.

СлЕДСТВИЕ. Произвольный пермутационный $G$-модуль представляется в виде

$$
\mathbb{F}_{p}\left(T, t^{0}\right)=\bigoplus_{x \in G \backslash T} M_{x},
$$

где $M_{x}=\left\langle O_{x}\right\rangle$ для G-орбить $O_{x} \subseteq T$. При этом $M_{x^{0}}=\{0\}$, если $x^{0}-$ орбита точки $t^{0}$, и $M_{x} \cong \mathbb{F}_{p}\left(G / G_{t(x)}\right)$ в остальных случаях, где $t(x)$ - какой-либо әлемент из орбить $O_{x}$.

(1.12) Лемма. (а) Пусть $H$ - подгруппа про-р-группь $G, M$ - некоторьй пермутационный $H$-модуль. Тогда индуцированный модуль $\mathbb{F}_{p}(G) \otimes_{H} M$ является пермутационным $G$-модулем.

(b) Пусть $A=\mathbb{F}_{p}\left(T, t^{0}\right)$ - пермутачионный $G$-модуль, $N \triangleleft G$. Тогда пространство $N$-орбит $N \backslash T$ мохет естественным образом рассматриваться как пунктированное $G / N$-пространство, и имеет место естественный изо-

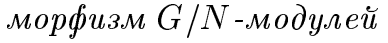

$$
A / I(N) A \cong \mathbb{F}_{p}\left(N \backslash T, y^{0}\right)
$$


ДоказАтельство. (а) Пусть $M=\mathbb{F}_{p}\left(T, t^{0}\right)$. Подмножество

$$
\widetilde{T}=\{g \otimes t \mid g \in G, t \in T\}
$$

является, как легко видеть, замкнутым $G$-подпространством модуля $\widetilde{M}=$ $\mathbb{F}_{p}(G) \otimes_{H} M$. Докажем, что вложение $(\widetilde{T}, 0)$ в $\mathbb{F}_{p}$-модуль $\widetilde{M}$ обладает универсальным свойством $(*)$ из (1.7).

Используя заданное отображением $g \otimes m \mapsto g H \otimes m, m \in M$, стандартное отождествление и перестановочность тензорного произведения с проективными пределами (см. [9]), разложим $\widetilde{M}$ в проективный предел

$$
\widetilde{M}=\mathbb{F}_{p}(G / H) \otimes M=\lim _{\longleftarrow} \widetilde{M}_{\lambda},
$$

где

$$
\widetilde{M}_{\lambda}=\mathbb{F}_{p}\left(G / V_{\lambda}\right) \otimes M=\bigoplus_{g V_{\lambda} \in G / V_{\lambda}}\left(\mathbb{F}_{p}\left(g V_{\lambda}\right) \otimes M\right)
$$

Здесь $V_{\lambda}, \lambda \in \Lambda$, пробегает все открытые подгруппы $G$, содержащие $H$. Ясно, что достаточно проверить свойство $(*)$ для образов $\left(\widetilde{T}_{\lambda}, 0\right)$ пространства $\widetilde{T}$ в модулях $\widetilde{M}_{\lambda}$. Это, однако, очевидно, так как $\widetilde{T}_{\lambda}$ равно объединению конечного числа подпространств $\left\{g V_{\lambda} \otimes t \mid t \in T\right\}, g V_{\lambda} \in G / V_{\lambda}$, из прямых слагаемых, изоморфных $M$ как $\mathbb{F}_{p}$-модули.

(b) Каноническое отображение $T$ на $N \backslash T$, рассматриваемое как $G$-отображение, определяет гомоморфизм $A$ на $\mathbb{F}_{p}\left(N \backslash T, y^{0}\right)$, являющийся, очевидно, $G$-модульным. Ядро $K$ этого гомоморфизма порождено как $\mathbb{F} p$-подмодуль элементами $\left(n_{1}-1\right) n_{2} t \in I(N) A$, где $t \in T, n_{1}, n_{2} \in N$. Обратное включение $I(N) A \subseteq K$ вытекает из тривиальности действия $N$ на $\mathbb{F}_{p}\left(N \backslash T, y^{0}\right)$.

(1.13) Для $G$-пространства $\left(T, t^{0}\right)$ положим

$$
\operatorname{ess}_{G}(T)=\left\{t \in T \mid G_{t} \neq\{1\}\right\}
$$

а через $\overline{\operatorname{ess}}_{G}(T)$ будем обозначать замыкание в $T$ подмножества $\operatorname{ess}_{G}(T)$. Ясно, что $t^{0} \in \operatorname{ess}_{G}(T)$ и, следовательно, $\left(\overline{\operatorname{ess}}_{G}(T), t^{0}\right)$ - пунктированное $G$-пространство.

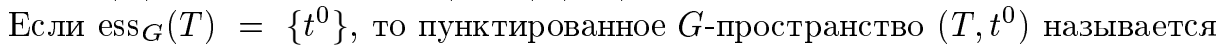
свободнылм. Не пунктированное $G$-пространство $S$ будем называть свободнылм,

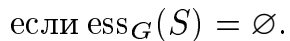

Через $T_{F}=T / \overline{\mathrm{ess}}_{G}(T)$ обозначим факторпространство, полученное стягиванием $\overline{\operatorname{ess}}_{G}(T)$ в точку $t_{F}^{0}$. Ясно, что $\left(T_{F}, t_{F}^{0}\right)$ является свободным пунктированньг $G$-пространством. В случае, когда пространство орбит $Y=G \backslash T_{F}$ бесконечно, его вес $w(Y)$ будем обозначать через $\operatorname{fr}_{G}\left(T, t^{0}\right)$. Если $Y$ конечно, то положим $\operatorname{fr}_{G}\left(T, t^{0}\right)=|Y|-1$.

(1.14) ПРЕДЛОЖЕНИЕ. Любой пермутационнъй G-модуль представляется в виде

$$
\mathbb{F}_{p}\left(T, t^{0}\right) \cong \mathbb{F}_{p}\left(\overline{\operatorname{ess}}_{G}(T), t^{0}\right) \oplus P,
$$

әде $P$ - свободный $G$-модуль ранга $\operatorname{fr}_{G}\left(T, t^{0}\right)$. 
ДокаЗАТЕЛЬСтво. По лемме (1.10) подмодуль $E=\left\langle\overline{\mathrm{ess}}_{G}(T)\right\rangle$ изоморфен $\mathbb{F}_{p}\left(\overline{\operatorname{ess}}_{G}(T)\right)$, а фактормодуль по нему изоморфен $\mathbb{F}_{p}\left(T_{F}, t_{F}^{0}\right)$. Из следствия (1.11) и свободы $G$-пространства $\left(T_{F}, t_{F}^{0}\right)$ вытекает, что $\mathbb{F}_{p}\left(T_{F}, t_{F}^{0}\right)$ является прямой суммой свободных $G$-модулей (рангов 1 и 0 ). Таким образом, $\mathbb{F}_{p}\left(T_{F}, t_{F}^{0}\right)-$ свободньй $G$-модуль (по лемме (1.6)) и $\mathbb{F}_{p}\left(T, t^{0}\right)=E \oplus P$, где $P \cong \prod_{i \in I} \mathbb{F}_{p}(G)$. По лемме (1.12.(b)) факторгруппа $P / I(G) P \cong \prod_{i \in I} \mathbb{Z} / p \mathbb{Z}$ изоморфна $\mathbb{F} p\left(G \backslash T_{F}, t_{F}^{0}\right)$. Отсюда в силу сделанного в (1.7) замечания получаем, что $|I|=\operatorname{fr}_{G}\left(T, t^{0}\right)$.

(1.15) Для удобства ссылок сформулируем два утверждения, вытекающих из предыдущего предложения. Первое из них позволяет достаточно широко варьировать базисное $G$-подпространство пермутационного модуля.

СлеДСтвИЕ. (а) Пусть $\left(T, t^{0}\right)$ - некоторое $G$-пространство, $V$ - произвольное проконечное пространство веса $w(V)=\operatorname{fr}_{G}\left(T, t^{0}\right)$. Тогда

$$
\mathbb{F}_{p}\left(T, t^{0}\right) \cong \mathbb{F}_{p}\left(T^{\prime}, t^{0}\right)
$$

әде $T^{\prime}$ - дизбюнктное объединение пунктированного G-пространства $\left(\overline{\operatorname{ess}}_{G}(T), t^{0}\right)$ и не пунктированного G-пространства $G \times V$ (с очевиднымм действием $G$ ).

(b) Произвольный свободный $G$-модуль $P$ является свободным как $H$-модуль для любой подгруппь $H$ про-р-группьь $G$.

ДокаЗАтЕльство. (а) Замечаем, что $\mathrm{fr}_{G}\left(T^{\prime}, t^{0}\right)=w(V)$, и применяем предложение (1.14).

(b) В силу утверждения (a) $P \cong \mathbb{F}_{p}(G \times V)$, где $w(V)=\operatorname{rank} P$. Свободное $G$-пространство $G \times V$ является свободным и как $H$-пространство. Поэтому из предложения (1.14) вытекает свобода $H$-модуля $\mathbb{F}_{p}(G \times V)$.

(1.16) Пусть $G$ - про- $p$-группа, $M$ - некоторьй $G$-модуль. Подмодуль $M_{0}$ называется сердиевиной $M$ (в записи, $\left.M_{0}=\operatorname{core}(M)\right)$, если справедливо следуюшее:

(i) фактормодуль $M / M_{0}$ является свободным;

(ii) $M_{0}$ не имеет фактормодулей, изоморфных $\mathbb{F}_{p}(G)$.

ПРЕДЛОЖЕНИЕ. Произвольный $G$-модуль $M$ обладает сердиевиной. Если $A=\operatorname{core}(M)$ и $B=\operatorname{core}(M)$, то $B=\alpha(A)$ для некоторого автоморфизма $\alpha$ модуля $M$.

ДокАЗАТЕльство. В качестве сердцевины модуля $M$ можно взять произвольньй минимальный по включению элемент $A$ множества $\Psi$ таких подмодулей $K$, что фактормодуль $M / K$ является свободньм. Возможность применения к $\Psi$ леммы Цорна вытекает из леммы (1.6.(а)). А именно, если $\left\{K_{j} \mid j \in J\right\}$-цепш в $\Psi$, то для пересечения $K=\bigcap_{j \in J} K_{j}$ фактормодуль $M / K=\lim M / K_{j}$ также является свободньм.

Свойство (ii) сердцевины $M$ для так определенного подмодуля $A$ является следствием его минимальности в $\Psi$. Действительно, если $A / A_{1} \cong \mathbb{F}_{p}(G)$, то фактормодуль $M / A_{1} \cong\left(A / A_{1}\right) \oplus(M / A)$ свободен.

Докажем второе утверждение. Пусть $M=A \oplus P$ и $M=B \oplus Q$ для свободных подмодулей $P$ и $Q$. Через $\delta_{A}$ и $\delta_{Q}$ обозначим ретракции $M$ на $A$ и на $Q$ в этих прямых разложениях, т.е. $\delta_{A}(a+p)=a, \delta_{Q}(b+q)=q$. Положим $\eta=\delta_{Q} \delta_{A}: M \rightarrow M$ и $\alpha=1-\eta$, т.е. $\alpha(m)=m-\eta(m)$ для $m \in M$. 
Докажем, что эндоморфизм $\alpha$ модуля $M$ является его автоморфизмом. Для этого достаточно показать, что бесконечная сумма

$$
\beta(m)=m+\eta(m)+\eta^{2}(m)+\eta^{3}(m)+\cdots,
$$

где $m \in M$, задает вполне определенный элемент $M$. Тогда, как легко видеть, отображение $m \mapsto \beta(m)$ будет обратным к $\alpha$.

Заметим сначала, что для любого открытого подмодуля $U$ в $G$-модуле $M$ из конечности модуля $M / U$ вытекает сушествование такого $n \geqslant 1$, что $I(G)^{n} M \subseteq U$. Поэтому для доказательства сушествования элемента $\beta(m)$ достаточно показать, что $\eta^{n}(M) \subseteq I(G)^{n} M$ для всех $n \geqslant 1$. Тривиальный в силу равенства $\eta\left(I(G)^{n} M\right)$ $=I(G)^{n} \eta(M)$ индуктивный переход позволяет ограничиться доказательством включения $\eta(M) \subseteq I(G) M$.

Из определения эндоморфизма $\eta$ следует, что $\eta(M) \subseteq Q$ и $\eta(P)=\{0\}$. Поэтому достаточно показать, что $\eta(A) \subseteq I(G) Q$.

Предположим, что $\eta(A) \nsubseteq I(G) Q$. Тогда, так как $Q / I(G) Q$ - тривиальный $G$-модуль, изоморфный $\prod_{i \in I} \mathbb{Z} / p \mathbb{Z}$, сушествует такой эпиморфизм $\xi: Q \rightarrow$ $\mathbb{F}_{p}(G) / I(G)$, что $\xi \eta(A) \neq\{0\}$. В силу проективности $Q$ эпиморфизм $\xi$ поднимается до гомоморфизма $\psi: Q \rightarrow \mathbb{F}_{p}(G)$. Тогда для $\varphi=\psi \eta$ образ $\varphi(A) \nsubseteq I(G)$ и, следовательно, $\varphi(A)=\mathbb{F}_{p}(G)$. Это, однако, противоречит свойству (iі) сердцевины $A$.

Итак, $\alpha$ - автоморфизм модуля $M$. Докажем, что $\alpha(A)=B$. Сначала заметим, что если $a=b+q \in A$, где $b \in B, q \in Q$, то $\eta(a)=\delta_{Q}(a)=q$. Следовательно, $\alpha(A) \subseteq B$. Отсюда и из прямого разложения $M=\alpha(A) \oplus \alpha(P)$ вытекает, что $B=\alpha(A) \oplus L$, где $L=B \cap \alpha(P)$.

Используя разложение $M=B \oplus Q$, получаем, что $L \oplus Q \cong M / \alpha(A) \cong P$. Таким образом, $L$ - свободный $G$-модуль. В силу свойства (ii) сердцевины $B$ имеем $L=\{0\}$, т.е. $B=\alpha(A)$.

(1.17) ПРЕДЛОЖЕНИЕ. Для $G$-пространства $\left(T, t^{0}\right)$ nодмодуль $\left\langle\overline{\operatorname{ess}}_{G}(T)\right\rangle \cong$ $\mathbb{F}_{p}\left(\overline{\operatorname{ess}}_{G}(T), t^{0}\right)$ является сердиевиной $\mathbb{F}_{p}\left(T, t^{0}\right)$.

ДокАЗАТЕЛЬСтво. В силу предложения (1.14) достаточно доказать, что не существует эпиморфизма $\varphi$ модуля $\mathbb{F}_{p}\left(\overline{\operatorname{ess}}_{G}(T), t^{0}\right)$ на свободный $G$-модуль $\mathbb{F}_{p}(G)$.

Предположим, что $\varphi$ - такой эпиморфизм. Так как $\mathbb{F}_{p}\left(\overline{\operatorname{ess}}_{G}(T), t^{0}\right)$ порождается подмножеством $\operatorname{ess}_{G}(T)$, то $\varphi(t) \notin I(G)$ для некоторой точки $t$, стабилизатор которой $G_{t} \neq\{1\}$. Поэтому $g \cdot \varphi(t)=\varphi(g \cdot t)=\varphi(t)$ для некоторого элемента $g \neq 1$. Это, однако, противоречит тому, что $\varphi(t)-$ свободньй порождающий $G$-модуля $\mathbb{F}_{p}(G)$.

(1.18) Два пермутационных $G$-модуля $\mathbb{F}_{p}\left(T, t^{0}\right)$ и $\mathbb{F}_{p}\left(V, v^{0}\right)$ назовем подобнылми, если $\mathbb{F}_{p}\left(\overline{\operatorname{ess}}_{G}(T), t^{0}\right) \cong \mathbb{F}_{p}\left(\overline{\operatorname{ess}}_{G}(V), v^{0}\right)$.

ПРЕДЛОЖЕНИЕ. Пусть $G$ - nро-p-гpупna, $M, K$ - maкue $G$-модули, что $M \oplus P \cong K \oplus Q$ для некоторых свободных $G$-модулей $P$ u $Q$. Тогда из пермутационности модуля $M$ следует, что $K$ - пермутационный модуль, подобный $M$. 
ДокаЗАТЕльство. Заметим сначала, что если $K_{0}=\operatorname{core}(K), M_{0}=\operatorname{core}(M)$, то $K_{0}=\operatorname{core}(K \oplus Q)$ и $M_{0}=\operatorname{core}(M \oplus P)$. В силу предположения $(1.16) K_{0} \cong M_{0}$. Если $M=\mathbb{F}_{p}\left(T, t^{0}\right)$, то из предложения $(1.17)$ следует, что $K \cong \mathbb{F}_{p}\left(\overline{\operatorname{ess}}_{G}(T), t^{0}\right) \oplus L$, где $L$ - свободный $G$-модуль. Из пермутационности свободных модулей вытекает теперь пермутационность $K$. Утверждение о подобии $M$ и $K$ немедленно следует из предложения (1.17).

\section{§ 2. Асферические группы: определение и примеры}

(2.1) Пусть $G$ - про-р-группа. Ее копредставлением будем называть эпиморфизм $\varphi: F \rightarrow G$, где $F$ - некоторая свободная про- $p$-группа. Часто мы будем отождествлять $G$ с факторгруппой $F / N$, где $N=\operatorname{Ker} \varphi$, и записывать копредставление в виде равенства $G=F / N$.

Копредставление $\varphi: F \rightarrow G$ называется минимальным, если $N=\operatorname{Ker} \varphi$ содержится в $F^{*}=F^{p}[F, F]$. В случае конечно порожденной группы $G$ минимальность $\varphi$ эквивалентна равенству $\operatorname{rank} F=d(G)$, где $d(G)$ - минимальное число порождающих $G, \operatorname{rank} F$ - ранг свободной про- $p$-группы $F$, равный, очевидно, $d(F)$.

Сопряжение в группе $F$ естественным образом преврашает факторгрупшу $\bar{N}=$ $N / N^{*}$, где $N^{*}=N^{p}[N, N]$, в левьй $\mathbb{F}_{p}(G)$-модуль. Везде ниже будем называть $\bar{N}$ модулем соотношений группь $G$, соответствующим копредставлению $\varphi$.

Так как $N^{*}$ является подгруппой Фраттини про- $p$-групшы $N$, то модуль соотношений $\bar{N}$ обладает следующим полезньм свойством.

Лемма. Для копредставления $G=F / N$ подмножество $R \subset N$ порождает $N$ как нормальный делитель в $F$ (т.е. является мнохеством определяющих соотношений $G$ для данного копредставления), если его образ $\bar{R}$ порождает $G$-модуль $\bar{N}$.

(2.2) ОПРЕДЕЛЕНИЕ. Про-р-группа $G$ называется асферической, если сушествует такое ее копредставление $G=F / N$, что модуль соотношений $\bar{N}$ является пермутационным $G$-модулем.

Стоит, вероятно, сразу же отметить, что из доказанной ниже теоремы (2.5) вытекает, что пермутационность одного из модулей соотношений про-p-групшы $G$ влечет пермутационность всех ее модулей соотношений.

(2.3) Лемма. Пусть $F$ - свободная про-р-группа с базисом $X, \varphi: F \rightarrow G$ - эпиморфизм, $N=\operatorname{Ker} \varphi$. Тогда существует точная последовательность G-модулей

$$
0 \longrightarrow \bar{N} \stackrel{\delta}{\longrightarrow} L_{X} \stackrel{\lambda}{\longrightarrow} \mathbb{F}_{p}(G) \stackrel{\varepsilon}{\longrightarrow} \mathbb{Z} / p \mathbb{Z} \longrightarrow 0
$$

әде $L_{X}-$ свободный $G$-модуль с базисом $\left\{w_{x} \mid x \in X\right\}, \lambda\left(w_{x}\right)=\varphi(x)-1$ и для $n \in N$

$$
\delta(\bar{n})=\sum_{x \in X} \varphi\left(\frac{\partial n}{\partial x}\right) w_{x}
$$

Здесь $\bar{n}$ - образ элемента $n \in N$ в факторгруппе $\bar{N}$, а элементы $\partial n / \partial x \in \mathbb{F}_{p}(F)$ являются про-р-аналогами производных Фокса [13]. Удобное их определение мы 
приведем ниже в доказательстве леммы (2.4). Сейчас же отметим, что отображение $\partial / \partial x$ из групшы $F$ в левый $F$-модуль $\mathbb{F}_{p}(F)$ можно охарактеризовать как непрерывный скрешенный гомоморфизм, заданньй равенствами $\partial x / \partial x=1$ и $\partial z / \partial x=1$ для всех $z \in X, z \neq x$.

В дальнейшем на (1) будем ссылаться как на точную последовательность модуля соотношений $\bar{N}$ про-р-групшы $G$.

Построение последовательности (1) проводится аналогично описанному в $\S 3.1$ [14] для случая дискретных групп. Выделим фрагмент рассуждений, содержащий необходимые в про-p-случае изменения, в виде следующей леммы.

(2.4) Пусть $H$ - подгруппа про-р-групшы $G$. Через $I_{G}(H)$ обозначим ядро гомоморфизма $\psi: \mathbb{F}_{p}(G) \rightarrow \mathbb{F}_{p}(G / H)$ левых $G$-модулей, индуцированное каноническим отображением $G$ на $G / H$. Ясно, что $I_{G}(H)=\mathbb{F}_{p}(G) I(H)$.

В случае, когда $H \triangleleft G$, левьй идеал $I_{G}(H)$ кольца $\mathbb{F}_{p}(G)$ является, очевидно, двусторонним. Это позволяет, в частности, получить равенство, $I_{G}(H)=$ $I(H) \mathbb{F}_{p}(G)$.

Лемма. Пусть $H$ - подгруппа свободной про-р-группь $F, Y-$ базис $H$ как свободной про-р-группь. Тогда $I_{F}(H)$ является левым свободным $F$-модулем с базисом $Y-1=\{y-1 \mid y \in Y\}$.

ДоказАтельство. (1) Рассмотрим сначала случай, когда $H=F$. Воспользуемся хорошо известным изоморфизмом $\gamma: \mathbb{F}_{p}(F) \rightarrow \mathbb{F}_{p}[[T]]$, где $\mathbb{F}_{p}[[T]]$ - кольцо формальных степенных рядов над $\mathbb{F}_{p}$ от множества $T=\left\{t_{y} \mid y \in Y\right\}$ некоммутирующих переменных (см., например, [4]). По построению $\gamma(y)=1+t_{y}$ для $y \in Y$.

Легко видеть, что идеал $\Delta(T)$, состоящий из рядов без свободного члена, является левым свободным $\mathbb{F}_{p}[[T]]$-модулем с базисом $T$. Так как $\gamma(I(F))=\Delta(T)$, то $F$-модуль $I(F)$ также свободен с базисом $Y-1$.

Обешанное выше явное определение свободных производных элемента $v \in F$ дается разложением

$$
v-1=\sum_{y \in Y} \frac{\partial v}{\partial y}(y-1)
$$

элемента $v-1 \in I(F)$ по базису $Y-1$.

(2) В случае, когда $H$ - произвольная подгруппа $F$, отметим как следствие части (1) доказательства, что $I(H)$ является свободньм $H$-модулем с базисом $Y-1$. Рассмотрим $\mathbb{F}_{p}(F)$ как правый $H$-модуль. По следствию (1.15.(b)) и лемме (1.4) этот модуль плоский. Поэтому вложение $I(H) \subset \mathbb{F}_{p}(H)$ индуцирует инъективнњй гомоморфизм левых $F$-модулей

$$
\chi: \mathbb{F}_{p}(F) \otimes_{H} I(H) \rightarrow \mathbb{F}_{p}(F) \otimes_{H} \mathbb{F}_{p}(H) \cong \mathbb{F}_{p}(F)
$$

образ которого совпадает с $\mathbb{F}_{p}(F) I(H)=I_{F}(H)$.

Так как модуль слева является, очевидно, свободным с базисом $\{1 \otimes(y-1) \mid$ $y \in Y\}$, то отсюда получаем, что $Y-1-$ свободный базис $F$-модуля $I_{F}(H)$.

ДокАЗАТЕЛЬСТво ЛЕммы (2.3). Факторы цепочки

$$
\mathbb{F}_{p}(F) \supset I(F) \supset I_{F}(N) \supset I_{F}(N) I(F)
$$


подмодулей левого $F$-модуля $\mathbb{F}_{p}(F)$ дают точную последовательность левых $F$-модулей

$0 \rightarrow I_{F}(N) / I_{F}(N) I(F) \rightarrow I(F) / I_{F}(N) I(F) \rightarrow \mathbb{F}_{p}(F) / I_{F}(N) \rightarrow \mathbb{F}_{p}(F) / I(F) \rightarrow 0$

на которых $N$ действует, очевидно, тривиально.

Докажем, что эта последовательность изоморфна (1) как последовательность $G$-модулей.

Ясно, что $\mathbb{F}_{p}(F) / I(F) \cong \mathbb{Z} / p \mathbb{Z}$ и эпиморфизм $\varphi$ дает изоморфизм $\mathbb{F}_{p}(F) / I_{F}(N)$ $\cong \mathbb{F}_{p}(G)$. По лемме $(2.4) I(F)$ - левый свободньй $F$-модуль с базисом $X-1$. Поэтому фактормодуль $I(F) / I_{F}(N) I(F)$ является левым свободным $G$-модулем, базис которого составляют образы $w_{x}$ элементов $x-1 \in X-1$.

Непосредственные вычисления показывают, что отображение $n \mapsto n-1$ из подгруппы $N \subseteq F$ в идеал $I_{F}(N)$ индуцирует гомоморфизм $\beta: \bar{N} \rightarrow I_{F}(N) / I_{F}(N) I(F)$ левых $G$-модулей. Докажем биективность $\beta$.

Пусть $Y$ - базис $N$ как свободной про-р-групшы. Используя правую версию леммы $(2.4)$, получаем, что $I_{F}(N) / I_{F}(N) I(F)$ является (правым) $\mathbb{F}_{p}$-модулем, свободным над образом $Y-1$. Так как $\bar{N}-$ свободный $\mathbb{F}_{p}$-модуль с базисом $\bar{Y}$ и $\beta$ биективно отображает базис на базис, то $\beta$ - изоморфизм $\mathbb{F}_{p}$-модулей.

(2.5) Для фиксированной про-р-групшы $G$ рассмотрим класс точных последовательностей левых $\mathbb{F}_{p}(G)$-модулей вида

$$
0 \rightarrow M \rightarrow P_{1} \rightarrow P_{0} \rightarrow \mathbb{Z} / p \mathbb{Z} \rightarrow 0
$$

в которых модули $P_{0}$ и $P_{1}$ свободны.

Подчеркнем, что все последовательности модулей соотношений группы $G$ принадлежат этому классу.

ТЕОРемА. (а) Если для про-р-группь $G$ существует последовательность (2), в которой модуль $M$ пермутационный, то $G$ - асферическая гpynna.

(b) Если $G$ - асферическая про-р-группа с пермутачионным модулем соотношений $\bar{N}$, то в любой последовательности (2) $M$ является пермутационнымм G-модулем, подобным $\bar{N}$.

ДокАЗАтЕльство. Применим лемму Шанюэля (см., например, [15]) к последовательности (2) и к последовательности (1) модуля соотношений $\bar{N}$ группы $G$. Получим изоморфизм

$$
M \oplus Q^{\prime} \cong \bar{N} \oplus Q^{\prime \prime}
$$

где $Q^{\prime}=L_{X} \oplus P_{0}, Q^{\prime \prime}=P_{1} \oplus \mathbb{F}_{p}(G)$ - свободные $G$-модули. В силу предложения (1.18) $G$-модули $M$ и $\bar{N}$ одновременно являются пермутационными и как пермутационные подобны. Отсюда, очевидно, вытекает справедливость обоих утверждений теоремы.

ЗАмЕчАниЕ. В дальнейшем будет доказано, что для асферической группы $G$ утверждение о подобии всех пермутационных модулей $M$, участвующих в последовательностях вида (2), усиливается до утверждения, что их базисные $G$-пространства по сушеству изоморфны (см. теорему (3.5.(b))). 
(2.6) ТЕОремА. (а) Класс асферических про-р-групп замкнут относительно образования подгрупп.

(b) Каждая про-р-группа $G$ когомологической размерности $\mathrm{cd} G \leqslant 2$ является асферической.

В частности, все свободные про-р-группь асферичны.

ДоказАтельство. (а) Пусть $G=F / N$ - асферическая группа, где $\bar{N}=$ $\mathbb{F}_{p}\left(T, t^{0}\right)$ для $G$-пространства $\left(T, t^{0}\right)$. Если $H$ - подгруппа $G$, то $H=L / N$ для свободной про- $p$-группы $L, N \subseteq L \subseteq F$. Таким образом, $\bar{N}$ - пермутационный модуль соотношений группы $H$ с базисньм $H$-пространством $\left(T, t^{0}\right)$.

(b) Условие $\mathrm{cd} G \leqslant 2$ эквивалентно, как известно, существованию свободной (в силу леммы (1.4)) $\mathbb{F}_{p}(G)$-резольвенты длины 2 модуля $\mathbb{Z} / p \mathbb{Z}$. Эта резольвента дает последовательность $G$-модулей вида (2) с пермутационньм по лемме (1.9) модулем $M$. Асферичность группы $G$ вытекает теперь из теоремы $(2.5)$.

(2.7) Ввиду утверждения (а) предыдущей теоремы следующую теорему можно рассматривать как описание класса конечных подгрупп асферических про-pгрупाг.

ТЕОРемА. Конечная р-группа является асферической тогда и только тогда, когда она ииклическая.

ДоказАтельство. (1) Пусть $G$ - конечная $p$-группа. Рассмотрим ее минимальное копредставление $G=F / N$. Так как ранг свободной про-ргруппы равен $d(G)<\infty$ и индекс $[F: N]=|G|<\infty$, то $d(N)<\infty$. Поэтому модуль соотношений $\bar{N}$ конечен. Известно (см., например, $[14 ; \S 11.7]$ ), что минимальньй модуль соотношений конечной $p$-группы прямо неразложим.

Если $G$ - асферическая группа, то по теореме $(2.5)$ конечньй модуль $\bar{N}$ является пермутационным. Из его неразложимости получаем в силу следствия (1.11), что $\bar{N} \cong \mathbb{F}_{p}(G / H)$ для некоторой подгруппы $H$.

Таким образом, размерность $\bar{N}$ над полем $\mathbb{F}_{p}$ равна $[G: H]=p^{m}, m \geqslant 0$. С другой стороны, из формулы Шрейера для ранга свободной подгруппы $N$ группы $F$ следует, что эта размерность равна $(d(G)-1) p^{k}+1$, где $p^{k}=|G|$, и поэтому не делится на $p$. Следовательно, $m=0$ и $d(G)=1$, т.е. $G$ - циклическая групша.

(2) Циклическая группа $G$ порядка $p^{n}$ имеет минимальное копредставление $G=$ $\mathbb{Z}_{p} / p^{n} \mathbb{Z}_{p}$. Соответствующий модуль соотношений изоморфен $\mathbb{Z} / p \mathbb{Z}$ и, следовательно, является пермутационным.

(2.8) Следуюшее утверждение дает, в частности, возможность строить новые асферические группы с помощью некоторых свободных конструкций в классе про-р-групп. Чтобы избежать громоздких топологических построений, приведем его здесь не в максимально возможной общности.

Будем говорить, что $\left(T, t^{0}\right)$ является $G$-пространством дискретного типа, если любая окрестность отмеченной точки $t^{0}$ содержит почти все $G$-орбиты пространства $\overline{\operatorname{ess}}_{G}(T)$. Это условие эквивалентно, очевидно, тому, что дополнение в пространстве орбит $G \backslash \overline{\operatorname{ess}}_{G}(T)$ отмеченной точки $G \cdot t^{0}=\left\{t^{0}\right\}$ является дискретным подпространством.

ТЕОРЕМА. Пусть для про-р-группь $G$ существует точная последовательность

$$
0 \longrightarrow \mathbb{F}_{p}\left(E, e^{0}\right) \stackrel{\varphi}{\longrightarrow} \mathbb{F}_{p}\left(V, v^{0}\right) \longrightarrow \mathbb{Z} / p \mathbb{Z} \longrightarrow 0
$$


пермутационных модулей, в которой

(i) $\left(E, e^{0}\right) u\left(V, v^{0}\right)-G$-пространства дискретного типа,

(ii) стабилизаторь $G_{v}$ точек $v \in V, v \neq v^{0}$, асферичны,

(iii) стабилизаторы $G_{e}$ точек $е \in E, e \neq e^{0}$, свободньи.

Тогда $G$ - асферическая группа.

ДоКАЗАТЕЛЬСТво основано на идеях, использованных в [16] при доказательстве предложения 6 в $\S 1$.

Применяя к каноническому отображению $\overline{\operatorname{ess}}_{G}(V)$ на $G \backslash \overline{\operatorname{ess}}_{G}(V)$ утверждение (а) леммы (1.10) и учитывая лемму (1.5), из предложения (1.14) получаем

$$
\mathbb{F}_{p}\left(V, v^{0}\right)=P \oplus\left(\prod_{\alpha \in A} \mathbb{F}_{p}\left(G / H_{\alpha}\right)\right)
$$

где $P$ - свободньй $G$-модуль, $A$ - дополнение в $G \backslash \overline{\operatorname{ess}}_{G}(V)$ точки $G \cdot v^{0}=\left\{v^{0}\right\}$, $H_{\alpha}$ - стабилизатор в $G$ какой-либо точки из $G$-орбиты $\alpha \in A$.

Для каждого $\alpha \in A$ возьмем последовательность

$$
0 \rightarrow M_{\alpha} \rightarrow L_{\alpha} \rightarrow K_{\alpha} \rightarrow \mathbb{Z} / p \mathbb{Z} \rightarrow 0
$$

модуля соотношений группы $H_{\alpha}$. По условию $M_{\alpha}$ - пермутационный $H_{\alpha}$-модуль. Умножив (4) тензорно над $H_{\alpha}$ на правый $H_{\alpha}$-модуль $\mathbb{F}_{p}(G)$, получим последовательность левых $G$-модулей

$$
0 \rightarrow \widetilde{M}_{\alpha} \rightarrow \widetilde{L}_{\alpha} \rightarrow \widetilde{K}_{\alpha} \rightarrow \mathbb{F}_{p}\left(G / H_{\alpha}\right) \rightarrow 0
$$

где $\widetilde{L}_{\alpha}$ и $\widetilde{K}_{\alpha}$ свободны, а $\widetilde{M}_{\alpha}=\mathbb{F}_{p}(G) \otimes_{H_{\alpha}} M_{\alpha}$ - пермутационный в силу леммы (1.12.(a)) $G$-модуль. Так как по следствию (1.15.(b)) и лемме (1.4) $H_{\alpha}$-модуль $\mathbb{F}_{p}(G)$ плоский, то все полученные последовательности точны.

Возьмем их прямое произведение по всем $\alpha \in A$ и прибавим точную последовательность

$$
0 \longrightarrow 0 \longrightarrow 0 \longrightarrow P \stackrel{\text { id }}{\longrightarrow} P \longrightarrow 0 \text {. }
$$

Получим точную последовательность $G$-модулей

$$
0 \rightarrow \widetilde{M} \rightarrow \widetilde{L} \rightarrow \widetilde{K} \rightarrow \mathbb{F}_{p}\left(V, v^{0}\right) \rightarrow 0
$$

со свободными модулями $\widetilde{L}$ и $\widetilde{K}$ и с пермутационным по лемме (1.9) модулем $\widetilde{M}$.

Теперь через $B$ обозначим дополнение в $G \backslash \overline{\operatorname{ess}}_{G}(E)$ точки $G \cdot e^{0}=\left\{e^{0}\right\}$. Пусть $F_{\beta}-$ стабилизатор в $G$ какой-либо точки из $G$-орбиты $\beta \in B$. Так как по условию сd $F_{\beta} \leqslant 1$, то существуют свободные $F_{\beta}$-резольвенты

$$
0 \rightarrow I\left(F_{\beta}\right) \rightarrow \mathbb{F}_{p}\left(F_{\beta}\right) \rightarrow \mathbb{Z} / p \mathbb{Z} \rightarrow 0
$$

Повторив проделанные выше построения, используя эти последовательности вместо (4), получим свободную $G$-резольвенту модуля $\mathbb{F}_{p}\left(E, e^{0}\right)$

$$
0 \rightarrow \widetilde{Q}_{1} \rightarrow \widetilde{Q}_{0} \rightarrow \mathbb{F}_{p}\left(E, e^{0}\right) \rightarrow 0
$$


Стандартным образом (см., например, [17]) поднимем гомоморфизм $\varphi$ из последовательности (3) $G$-модулей до морфизма $\Phi=\left(\varphi_{2}, \varphi_{1}, \varphi_{0}\right)$ комплекса (6) в комплекс (5); здесь $\varphi_{2}=0$. Конус этого морфизма $\Phi$ (см. [17]) дает точную последовательность $G$-модулей

$$
0 \rightarrow \widetilde{M} \oplus \widetilde{Q}_{1} \rightarrow \widetilde{L} \oplus \widetilde{Q}_{0} \rightarrow \widetilde{K} \rightarrow \mathbb{Z} / p \mathbb{Z} \rightarrow 0
$$

в которой модули $\widetilde{K}$ и $\widetilde{L} \oplus \widetilde{Q}_{0}$ свободны, а $\widetilde{M} \oplus \widetilde{Q}_{1}-$ пермутационный $G$-модуль. Применяя теорему $(2.5)$, заключаем, что $G$ - асферическая группа.

(2.9) Применим предыдущую теорему для доказательства асферичности простейших свободных конструкций.

Свободное амальгамированное про-р-произведение $G=H * A K$ нам удобно будет считать факторгруппой $L / M, L=H * K$ - свободное про-р-произведение, $M$ - нормальная подгруппа в $L$, порожденная элементами $a^{-1} \beta(a)$, где $a$ пробегает подгруппу $A \leqslant H, \beta$ - изоморфизм $A$ на подгруппу $B \leqslant K$. Аналогично, HNN-расширение понимаем как факторгруппу $L / M, L=H *\langle t\rangle,\langle t\rangle \cong \mathbb{Z}_{p}$, а нормальная подгруппа $M$ порождена элементами $\operatorname{tat}^{-1} \beta(a)^{-1}$, где $a \in A \leqslant H$, $\beta$ - изоморфизм $A$ на подгруппу $B \leqslant H$.

Условимся говорить, что про-р-группа $G$ является амальгамированным произведением (или HNN-расширением) своих подгруп, если канонический эпиморфизм группы $L$ на $G$ инъективно отображает $H$ и $K$ в $G$. Заметим, что, как показано в [18], эта инъективность не всегда (в отличие от случая дискретных групп) имеет место.

СЛЕДСТВИЕ. Пусть $G$ - амальгамированное свободное про-р-произведение своих асферических подгрупп $H$ и $K$ или $\mathrm{HNN}$-расширение асферической подгруппь $H$. Тогда $G$ - асферическая про-р-группа при условии, что обвединяемая подгруппа $A$ или ассоциированные подгруппьи $A, B$ (в случае HNN-расширения) являются свободными.

ДокАЗАтЕльство. В обоих случаях $G$ - фундаментальная про- $p$-группа конечного графа групп $(\mathscr{G}, \Gamma)$. Граф Г состоит из одного ребра $е$ и либо двух вершин $v, w$, либо (в случае HNN-расширения) одной вершины $v$; при этом $\mathscr{G}(e)=A$, $\mathscr{G}(v)=H, \mathscr{G}(w)=K$. В предложении (3.8) статьи [7] построено стандартное про-р-дерево $S=S(\mathscr{G}, \Gamma)$ с действием $G$ на нем. Соответствующий цепной комплекс над $\mathbb{F}_{p}$ дает точную последовательность (3) с $G$-пространствами $E=G / A$ и $V=(G / H) \cup(G / K)$ или $V=G / H$ (см. нижнюю строку диаграммы из доказательства (3.8) в [7]). Ясно, что условия теоремы (2.8) выполнены.

(2.10) Из предыдушего следствия вытекает, разумеется, асферичность свободного про- $p$-произведения конечного числа асферических груп. Можно показать, что то же верно для так называемого ограниченного свободного про- $p$-произведения (определение см., например, в [19]) произвольного множества групп.

В настоящее время автору неизвестно, является ли асферическим произвольное свободное про- $p$-произведение семейства асферических групп, непрерывно индексированных точками проконечного пространства, введенное в [5] (а также, в иной форме, в [20]). 
Тем не менее, в отдельных случаях можно, применяя специальные приемы, установить асфееричность свободных про-p-произведений. В качестве примера рассмотрим конструкцию, которую назовем здесь свободной про-p-степенью $\star_{T} H$ группы $H$ над проконечным пространством $T$.

Проекция $H \times T$ на $T$ определяет непрерьвное разбиение $H \times T$ на слои $H_{t}=$ $\{(h, t) \mid h \in H\}$, которые для всех $t \in T$ можно считать группами, изоморфньми $H$. Непрерывное отображение $\chi: H \times T \rightarrow K$, где $K$ - проконечная групша, будем называть послойным гомоморфизмом, если $\chi\left(h_{1} h_{2}, t\right)=\chi\left(h_{1}, t\right) \chi\left(h_{2}, t\right)$.

По определению про-р-группа $G=\star_{T} H$, если существует такой послойный гомоморфизм $\omega: H \times T \rightarrow G$, что для любого послойного гомоморфизма $\chi: H \times$ $T \rightarrow K$, где $K-$ про- $p$-группа, существует единственный гомоморфизм $\varphi: G \rightarrow K$, обладающей свойством $\varphi \omega=\chi$.

(2.11) СлЕДСТВИЕ. Свободная про-р-степень $G=\star_{T} H$ асферической группы $H$ над проконечным пространством $T$ является асферической группой.

ДокАЗАТЕЛЬСтво основано на том, что группа $G$ изоморфна подгруппе асферической в силу (2.9) групшы $W=H * F(T)$, где $F(T)$ - свободная про- $p$-группа над пространством $T$ в смысле [12].

Через $U$ обозначим нормальную подгруппу в $W$, порожденную свободным сомножителем $H$. Несложно проверить, что $U \cong \star_{F(T)} H$ (см., например, [21; лемма 2.3]). Пусть $\omega: H \times F(T) \rightarrow U-$ соответствующий универсальный послойный гомоморфизм. В силу [12] можем считать, что $T \subset F(T)$. Тогда, применяя теорему (1.4) из [5], получаем, что подгруппа $U$, порожденная подмножеством $\omega(H \times T)$, изоморфна $G$.

\section{§ 3. Основные свойства асферических групп}

(3.1) Теорема. Пусть $G$ - асферическая про-р-группа, $\left(T, t^{0}\right)-$ такое $G$-пространство, что для некоторого копредставления $G=F / N$ модуль соотношений $\bar{N}$ изоморфен $\mathbb{F}_{p}\left(T, t^{0}\right)$. Тогда

(a) стабилизатор $G_{t}$ произвольной точки $t \in T$, отличной от $t^{0}$, является конечной ииклической группой;

(b) если $K$ - конечная подгруппа $G$, то $K \subseteq G_{t}$ для некоторой точки $t \neq t^{0}$ uз $T$

(c) $G_{t} \cap G_{s}=\{1\}$ для любих отличных от $t^{0}$ точек $t \neq s$ из $T$.

ДокАЗАТЕЛЬСтво. Последовательность модуля соотношений для $\bar{N}$ дает точную последовательность

$$
0 \longrightarrow \mathbb{F}_{p}\left(T, t^{0}\right) \stackrel{\delta}{\longrightarrow} P_{1} \stackrel{\lambda}{\longrightarrow} P_{0} \stackrel{\varepsilon}{\longrightarrow} \mathbb{Z} / p \mathbb{Z} \longrightarrow 0
$$

со свободными $G$-модулями $P_{0}$ и $P_{1}$, ранги которых равны 1 и $\operatorname{rank} F$ соответственно.

Образ $t \neq t^{0}$ при $\delta$ является ненулевым элементом из $P_{1}^{G_{t}}$. Поэтому в силу следствия (1.15.(b)) и леммы (1.3) группа $G_{t}$ конечна. Утверждение (a) вытекает теперь из теоремы $(2.7)$. 
По той же теореме (2.7) циклической является произвольная конечная подгруппа $K$ группы $G$. Если $K \neq\{1\}$, то модуль соотношений ее минимального копредставления $K \cong \mathbb{Z}_{p} / p^{m} \mathbb{Z}_{p}$ изоморфен $\mathbb{Z} / p \mathbb{Z}$. С другой стороны, $\bar{N}$ также является модулем соотношений группы $K$. Из теоремы (2.5.(b)) получаем, что если $\left(T, t^{0}\right)$ рассматривать как $K$-пространство, то $\mathbb{F}_{p}\left(\overline{\operatorname{ess}}_{K}(T), t^{0}\right) \cong \mathbb{Z} / p \mathbb{Z}$. Следовательно, $\overline{\mathrm{ess}}_{K}(T)=\left\{t, t^{0}\right\}$. Таким образом, в $T$ сушествует, причем единственная, точка $t \neq t^{0}$, неподвижная при действии $K$.

Из существования такой точки $t$ вытекает, что $K \subseteq G_{t}$. Из утверждения о единственности следует, что если $K=G_{t} \cap G_{s} \neq\{1\}$ при $t \neq t^{0} \neq s$, то $t=s$.

(3.2) ТЕОРемА. Пусть $G=F / N$ - асферическая про-р-группа, модуль соотношений $\bar{N}$ которой является пермутационным с базисным $G$-пространством $\left(T, t^{0}\right) \subset \bar{N}$. Тогда для каждой точки $t \in T, t \neq t^{0}$, существует такой әлемент $r_{t} \in N$, что

(a) образ $r_{t}$ в факторгруппе $\bar{N}=N / N^{*}$ coвпадает с $t$;

(b) стабилизатор $G_{t}$ точки $t$ равен образу в $G$ чентрализатора $C_{F}\left(r_{t}\right)$;

(c) $r_{t}=h_{t}^{q_{t}}$ для некоторого әлемента $h_{t} \in F$, порожсдающего подгруппу $C_{F}\left(r_{t}\right) \cong \mathbb{Z}_{p}, u q_{t}=\left|G_{t}\right|$.

(3.3) Отложив на время доказательство, покажем, как сфформулированная во введении теорема В выводится из двух предыдущих теорем.

Пусть $\left\{O_{i} \mid i \in I\right\}$ - множество всех $G$-орбит $T$, отличных от $G \cdot t^{0}=\left\{t^{0}\right\}$. Для каждого $i \in I$ выберем точку $t(i) \in O_{i}$. Через $h_{i}$ обозначим элемент $h_{t(i)}$ из теоремы (3.2). Пусть $S_{i}$ - подгруппа в $G$, порожденная образом элемента $h_{i}$, $r_{i}=h_{i}^{q_{i}}$, где $q_{i}=\left|S_{i}\right|$.

Из теоремы (3.2) следует, что $S_{i}=G_{t(i)}$. Теперь, учитывая (3.2.(a)), из следствия (1.11) получаем, что для $G \backslash T=I \cup\{*\}$ (где $\left.*=\left\{t^{0}\right\}\right) \bar{N}=\bigoplus_{x \in G \backslash T} A_{x}$, где $A_{i}$ - циклический $G$-подмодуль, порожденный элементом $\bar{r}_{i}=t(i)$, и сушествует канонический изоморфизм $A_{i} \cong \mathbb{F}_{p}\left(G / S_{i}\right)$, а $A_{*}=\{0\}$.

Таким образом, утверждения (a) и (b) теоремы В получены. Для доказательства (c) привлекаем теорему (3.1). Из утверждения (b) этой теоремы следует, что $K \subseteq G_{t}$ для некоторого $t \in T, t \neq t^{0}$. Если $t \in O_{i}$, то $t=g \cdot t(i)$ и, следовательно, $G_{t}=g S_{i} g^{-1}$. Если кроме того $K \subseteq h S_{j} h^{-1}=G_{v}$, где $v=h \cdot t(j)$, то $v=t$ по (3.1.(c)). Поэтому $j=i$ и $g^{-1} h \in G_{t(i)}=S_{i}$.

ЗАмЕчАниЕ. Стремление придать теореме В традиционную форму привело к некоторой потере информации о строении $\bar{N}$ как пермутационного $G$-модуля. Эта информация восстанавливается, если дополнить формулировку теоремы В следующим утверждением:

(d) әлементыл $r_{i} \in N$ могут бить выбраны так, что подмнохсество

$$
T=\left(\bigcup_{i \in I} G \cdot \bar{r}_{i}\right) \cup\{0\}
$$

замкнуто в G-модуле $\bar{N}$. 
(3.4) ЛЕммА. Пусть $F-$ свободная про-р-группа, $N \triangleleft F, a-$ әлемент из $F$, образ которого в $F / N$ имеет конечный порядок $q=p^{m}>1$. Тогда $a^{q} \notin N^{*}$ u, более того, свободная про-р-группа $N$ имеет базис вида $Y=\left\{a^{q}, a^{k} y_{j} a^{-k}\right.$ $j \in J, 0 \leqslant k \leqslant q-1\}$ для некоторого мнохсества әлементов $y_{j} \in N$.

ДоказАТЕЛЬство. Без ограничения общности можем считать, что $F=\langle a, N\rangle$. Так как $a \notin N$, то $a \notin F^{*}$. Тогда $F / F^{*}=\langle\beta(a)\rangle \times B$, где $\beta$ - канонический эпиморфизм $F$ на $F / F^{*}$ и $B \subseteq \beta(N)$. Это позволяет построить свободный базис $F$ вида $\left\{a, y_{j} \mid j \in J\right\}$, где $y_{j} \in N$. Нетрудно видеть, что в силу открытости подгруппы $N$ в $F$ для построения свободного базиса групшы $N$ можно использовать переписывающий процесс Рейдемейстера-Шрейера. Для системы представителей $\left\{a^{k} \mid 0 \leqslant k \leqslant q-1\right\}$ он приводит к множеству $Y$.

ДокАЗАТЕЛЬСТво ТЕоремы (3.2). Так как нам будет важна $G$-модульная структура $\bar{N}$, условимся записывать аддитивно эту факторгруппу мультипликативной группы $N$. Пусть $\varphi: F \rightarrow G$ и $\beta: N \rightarrow \bar{N}$ - канонические эпиморфизмы.

Если для $t \in T, t \neq t^{0}$, стабилизатор $G_{t}=\{1\}$, то в качестве $h_{t}=r_{t}$ можем взять произвольный прообраз при $\beta$ элемента $t \in \bar{N}$. Поэтому в дальнейшем будем считать, что $q=\left|G_{t}\right|>1$.

Пусть $a$ - такой элемент из $F$, что $G_{t}=\langle\varphi(a)\rangle$. Нужный в теореме элемент $h_{t}$ построим в виде $h=n a^{k}$, где $n \in N, 1 \leqslant k \leqslant p-1$.

Из леммы (3.4) следует, что $\bar{N}$, рассматриваемый как $G_{t}$-модуль, имеет вид $\bar{N}=\langle b\rangle \oplus W$, где $b=\beta\left(a^{q}\right), W-$ свободный $G_{t}$-модуль. Пусть элемент $t \in \bar{N}$ представляется в виде $t=c+v$, где $c=k b$ для $0 \leqslant k \leqslant p-1, v \in W$. Тогда из $t \in \bar{N}^{G_{t}}, c \in \bar{N}^{G_{t}}$ следует, что $v \in \bar{N}^{G_{t}}$. Поэтому $v$ не содержится ни в каком свободном базисе $G_{t}$-модуля $W$ и, следовательно, $v \in I\left(G_{t}\right) W$.

В частности, $v \in I(G) \bar{N}$. С другой стороны, из леммы (1.12.(b)) следует, что точка $t \neq t^{0}$ из $T$ не содержится в $I(G) \bar{N}$. Поэтому $c \neq 0$ и, следовательно, $(k, p)=1$. Таким образом, положив $d=a^{k}$, получаем $\beta(d)=c$ и элемент $s=\varphi(d)$ порождает подгруппу $G_{t}$.

Далее, нетрудно показать, что фундаментальный идеал $I\left(G_{t}\right)$ циклической $p$-группы $G_{t}=\langle s\rangle$ порядка $q$ порождается как $\mathbb{F}_{p}$-модуль элементом $1+s+s^{2}+$ $\cdots+s^{q-1}$. Следовательно,

$$
v=\left(1+s+s^{2}+\cdots+s^{q-1}\right) w
$$

для некоторого $w \in W \subset \bar{N}$. Пусть $w=\beta(n)$ для $n \in N$. Положим $h=n d$. Тогда, во-первых, его образ $\varphi(h)=s$ порождает $G_{t}$. Во-вторых, элемент $r=h^{q}$ принадлежит $N$ и может быть представлен в виде

$$
r=n\left(d n d^{-1}\right)\left(d^{2} n d^{-2}\right) \cdots\left(d^{q-1} n d^{-(q-1)}\right) d^{q} .
$$

Следовательно, $\beta(r)=v+c=t$.

Таким образом, осталось только проверить, что $\langle h\rangle=C_{F}(r)$. Легко показать, что централизатор $C_{F}(r)$ изоморфен $\mathbb{Z}_{p}$ (так как он является свободной про-pгруппой с нетривиальным центром). Ясно, что $\langle h\rangle \subseteq C_{F}(r)$ и что из неравенства $\langle h\rangle \neq C_{F}(r)$ вытекает сушествование такого $g \in C_{F}(r)$, что $h=g^{p}$. Но тогда $G_{t}=\langle\varphi(h)\rangle$ оказалось бы подгруппой индекса $p$ в подгруппе $K=\langle\varphi(g)\rangle$ группы $G$, что невозможно в силу утверждений (b) и (c) теоремы (3.1). 
(3.5) Используем теорему (3.2) для получения дополнительной информации о базисных $G$-пространствах модулей соотношений асферических про-p-групп $G$.

Элементы $g_{1}$ и $g_{2}$ про-р-группы $G$ будем считать әквивалентными, если $\left\langle g_{1}\right\rangle=\left\langle g_{2}\right\rangle$. Факторпространство замкнутого в $G$ подмножества

$$
G[p]=\left\{g \in G \mid g^{p}=1\right\}
$$

по этому отношению эквивалентности совпадает, очевидно, с факторпространстBOM

$$
\mathscr{S}_{p}(G)=G[p] / \mathbb{F}_{p}^{*}
$$

по действию $g \mapsto g^{\lambda}$ (для $\lambda=1,2, \ldots, p-1$ ) мультипликативной группы поля $\mathbb{F} p$. Иначе, $\mathscr{S}_{p}(G)$ - пространство подгрупп $G$ порядка не более $p$, снабженное стандартной топологией.

Сопряжение в группе $G$ превращает пунктированное пространство $\left(\mathscr{S}_{p}(G),\{1\}\right)$ в $G$-пространство.

TеоремА. Пусть $G$ - асферическая про -р -группа, $\left(T, t^{0}\right)$ - такое $G$-nространство, что $\bar{N} \cong \mathbb{F}_{p}\left(T, t^{0}\right)$ для некоторого копредставления $G=F / N$. Тогда

(a) подмножсество $\operatorname{ess}_{G}(T)$ замкнуто в $T$;

(b) пунктированные $G$-пространства $\left.\operatorname{ess}_{G}(T), t^{0}\right)$ u $\left(\mathscr{S}_{p}(G),\{1\}\right)$ изоморфHbl.

ДокАЗАТЕЛьство. Обозначим $E=F / N^{*}$ и будем считать $\bar{N}=N / N^{*}$ подгруппой $E$, содержашей $G$-подпространство $T$. При этом отмеченная точка $t^{0}$ совпадает с единицей. Канонический эпиморфизм $E$ на факторгрупшу $G=E / \bar{N}$ будем обозначать через $\psi$.

(1) Через $\rho: E \rightarrow E$ обозначим отображение $y \mapsto y^{p}$. Так как $\rho(E)$ замкнуто в $E$, то для доказательства утверждения (а) теоремы достаточно установить равенство

$$
\operatorname{ess}_{G}(T)=T \cap \rho(E)
$$

Если $y^{p}=t \in T$ и $t \neq 1$, то $\psi(y) \neq 1$. Ясно, что $\psi(y) \in G_{t}$ и, следовательно, $t \in \operatorname{ess}_{G}(T)$. Так как $1=t^{0} \in \operatorname{ess}_{G}(T)$, то получаем включение $T \cap \rho(E) \subseteq \operatorname{ess}_{G}(T)$. Обратное включение вытекает из того, что по теореме $(3.2) \operatorname{ess}_{G}(T) \subseteq \rho(E)$.

(2) Для доказательства утверждения (b) теоремы введем подмножество $S=$ $\rho^{-1}\left(\operatorname{ess}_{G}(T)\right)$. Из замкнутости $\operatorname{ess}_{G}(T)$ в $E$ следует компактность пространства $S$. Кроме того, $\rho(S)=\operatorname{ess}_{G}(T)$.

Докажем, что для элементов $s_{1}, s_{2} \in S$ равенство $\rho\left(s_{1}\right)=\rho\left(s_{2}\right)$ эквивалентно равенству $\psi\left(s_{1}\right)=\psi\left(s_{2}\right)$.

Пусть $\psi\left(s_{1}\right)=\psi\left(s_{2}\right)=g$. Если $g=1$, то $s_{i} \in \bar{N}=\operatorname{Ker} \psi$ и, следовательно, $s_{1}^{p}=1=s_{2}^{p}$. Если $g \neq 1$, то по лемме $(3.4) t_{i}=s_{i}^{p} \neq 1, i=1,2$. Так как, очевидно, $g \in G_{t_{1}} \cap G_{t_{2}}$, то равенство $t_{1}=t_{2}$ вытекает из теоремы (3.1.(c)).

Предположим теперь, что $s_{1}^{p}=s_{2}^{p}=t \in T$. Если $t=t_{0}=1$, то по лемме (3.4) $s_{i} \in \bar{N}, i=1,2$, и, следовательно, $\psi\left(s_{1}\right)=1=\psi\left(s_{2}\right)$. Если $t \neq 1$, то $\psi\left(s_{1}\right)$ и $\psi\left(s_{2}\right)$ являются элементами порядка $p$, содержащимися в стабилизаторе $G_{t}$. Так как $t \neq t^{0}$, то в силу теоремы (3.1.(а)) $G_{t}$ - циклическая $p$-группа. Следовательно, $s_{2}=s_{1}^{k} n$, где $1 \leqslant k \leqslant p-1, n \in \bar{N}=\operatorname{Ker} \psi$. Покажем, что $k=1$. 
Через $\eta$ обозначим канонический эпиморфизм $E$ на $E /[E, \bar{N}]$. Используя в нужном месте центральность подгруппы $\eta(\bar{N})$ в группе $\eta(E)$, получаем следующую цепочку равенств:

$$
\eta\left(s_{2}^{p}\right)=\eta\left(s_{1}^{k} n\right)^{p}=\eta\left(s_{1}^{k}\right)^{p} \eta(n)^{p}=\eta\left(s_{1}^{p}\right)^{k} .
$$

Замечаем теперь, что в модульных обозначениях $\eta(\bar{N})=\bar{N} / I(G) \bar{N}$. Поэтому в силу леммы (1.12.(b)) $\eta(\bar{N})$ является свободным $\mathbb{F}_{p}$-модулем над пространством $\left(\eta(T), \eta\left(t^{0}\right)\right)$. Отсюда, очевидно, следует, что для $t \neq t^{0}$ полученное выше равенство $\eta(t)=\eta(t)^{k}$ возможно только при $k=1$.

(3) Из доказанного выше утверждения следует, что пространства $\operatorname{ess}_{G}(T)=$ $\rho(S)$ и $\psi(S) \subseteq G[p]$ гомеоморфны одному и тому же факторпространству компактного пространства $S$. Явно гомеоморфизм

$$
\beta: \operatorname{ess}_{G}(T) \rightarrow \psi(S)
$$

задается равенством $\beta(t)=\psi(s)$, где $s \in S$ и $s^{p}=t$.

Покажем теперь, что каноническая сюръекция $\gamma: G[p] \rightarrow \mathscr{S}_{p}(G)$ биективно отображает $\psi(S)$ на $\mathscr{S}_{p}(G)$.

Ясно, что $\gamma^{-1}(\{1\})=\{1\}$ и $1 \in \psi(S)$. Если $\langle g\rangle \in \mathscr{S}_{p}(G)$ имеет порядок $p$, то по теореме (3.1) существует точка $t \neq t^{0}$ из $\operatorname{ess}_{G}(T)$, для которой $\langle g\rangle \subseteq G_{t}$. Пусть $s-$ такой элемент из $S$, что $\beta(t)=\psi(s)$. Тогда $\psi(s)$ - элемент порядка $p$ в циклической группе $G_{t}$ и, следовательно, $\langle\psi(s)\rangle=\langle g\rangle$, т.е. $\gamma(\psi(s))=\langle g\rangle$.

Предположим, что $\gamma\left(\psi\left(s_{1}\right)\right)=\langle g\rangle$. Тогда $1 \neq \psi\left(s_{1}\right) \in G_{t} \cap G_{t_{1}}$, где $t_{1}=s_{1}^{p}$. По теореме (3.1.(c)) $t_{1}=t$. Следовательно, $\psi\left(s_{1}\right)=\beta\left(t_{1}\right)=\beta(t)=\psi(s)$.

(4) Осталось лишш проверить, что гомеоморфизм $\gamma \beta$ является $G$-отображением пространства $\left(\operatorname{ess}_{G}(T), t^{0}\right)$ на пространство $\left(\mathscr{P}_{p}(G),\{1\}\right)$. Пусть $h=\psi(w) \in G$, $t=s^{p} \in \operatorname{ess}_{G}(T)$ для некоторых $w \in E, s \in S$. Тогда $h \cdot t=w t w^{-1}=\left(w s w^{-1}\right)^{p}$ и $h\langle\psi(s)\rangle h^{-1}=\left\langle\psi\left(w s w^{-1}\right)\right\rangle$.

(3.6) Приведем одно следствие из теоремы (3.1), которое дает обратное к теореме (2.6.(b)) утверждение.

СлЕДСТВИЕ. Если $G$ - асферическая про-р-группа без кручения, то $\operatorname{cd} G \leqslant 2$.

ДокАЗАтЕльство. Пусть $G=F / N$ - копредставление с пермутационньм модулем $\bar{N}=\left(T, t^{0}\right)$. По теореме (3.1.(а)) стабилизаторы всех точек $t \neq t^{0}$ из $T$ тривиальны, т.е. $\operatorname{ess}_{G}(T)=\left\{t^{0}\right\}$. Тогда из предложения (1.14) следует, что $\bar{N}-$ свободньй $G$-модуль. Поэтому последовательность модуля соотношений является свободной $G$-резольвентой длины 2 модуля $\mathbb{Z} / p \mathbb{Z}$, откуда $\mathrm{cd} G \leqslant 2$.

(3.7) Следующая теорема дает характеризацию в терминах модулей соотношений класса асферических про-p-групп $G$, имеющих виртуальную когомологическую размерность vсd $G \leqslant 2$.

Будем говорить, что $G$-пространство $\left(T, t^{0}\right)$ имеет компактный mun, если отмеченная точка $t^{0}$ является изолированной в подпространстве $\overline{\operatorname{ess}}_{G}(T)$.

ТЕОРемА. Пусть $G$ - асферическая про-р-группа с модулем соотноиений $\bar{N}=\mathbb{F}_{p}\left(T, t^{0}\right)$. Группа $G$ тогда и только тогда содержит открытую подгруппу $H$ когомологической размерности $\mathrm{cd} H \leqslant 2$, когда $\left(T, t^{0}\right)$ является G-пространством компактного типа. 
ДоКАЗАТЕЛЬСТво. Воспользуемся гомеоморфизмом пунктированных пространств

$$
\beta:\left(\operatorname{ess}_{G}(T), t^{0}\right) \rightarrow(\psi(S), 1)
$$

из доказательства теоремы (3.5). Если точка $t^{0}$ изолирована в $\operatorname{ess}_{G}(T)$, то $\{1\}-$ открытое подмножество в $\psi(S)$. Следовательно, $\psi(S) \cap H=\{1\}$ для некоторой открытой подгруппы $H$ группы $G$. Предположим, что существует элемент $g \in H$ порядка $p$. Тогда, как показано в части (3) доказательства теоремы (3.5), $\langle g\rangle=\langle\psi(s)\rangle$ для некоторого $s \in S$. Ясно, что $1 \neq \psi(s) \in H$.

Из полученного противоречия следует, что асферическая группа $H$ не имеет кручения. Поэтому в силу следствия (3.6) cd $H \leqslant 2$.

Обратно, пусть $H$ - открытая подгруппа $G$ без кручения. Так как $\psi(S) \subseteq G[p]$, то $H \cap \psi(S)=\{1\}$. Поэтому подмножество $\{1\}$ открыто в $\psi(S)$, что эквивалентно изолированности $t^{0}$ в $T$.

(3.8) Теорема А, сформулированная во введении, является, в сущности, лишш переформулировкой теоремы (3.7).

В самом деле, если для копредставления $G=F / N$ модуль $\bar{N}=\mathbb{F}_{p}(S)$, где $S$ - не пунктированное $G$-пространство, то $\bar{N}=\mathbb{F}_{p}\left(T, t^{0}\right)$, где $T=S \cup\left\{t^{0}\right\}$ с изолированной отмеченной точкой $t^{0}$. Обратно, предположим, что $\bar{N}=\mathbb{F}_{p}\left(T, t^{0}\right)$ и точка $t^{0}$ изолирована в $\operatorname{ess}_{G}(T)$. Тогда $\bar{N}=\mathbb{F}_{p}\left(T^{\prime}, t^{0}\right)$, где $T^{\prime}-G$-пространство из следствия (1.15.(a)). Из его описания следует, что точка $t^{0}$ изолированаво всем $T^{\prime}$. Поэтому $\bar{N} \cong \mathbb{F}_{p}(S)$ для $S=T^{\prime}-\left\{t^{0}\right\}$.

\section{§4. Разрешимые нормальные подгруппы и центр}

В этом параграфе исследуется структура асфферической группы $G$ с абелевой нормальной подгруппой $A \neq\{1\}$. Группа $A$ обязана быть изоморфной одной из групп $\mathbb{Z} / p^{m} \mathbb{Z}, \mathbb{Z}_{p}$ или $\mathbb{Z}_{p} \times \mathbb{Z}_{p}$ (см. следствие (4.2)). Наибольший интерес представляет случай $A \cong \mathbb{Z}_{p}$, рассматриваемый в теореме (4.5); в остальных случаях группа $G$ оказьвается разрешимой (предложение (4.1) и теорема (4.4)). Особо хотелось бы отметить утверждение (b) теоремы (4.5) о локальном строении группы $G$. Ситуация теоремы (4.5) возникает в теореме (4.6) о центре и теореме (4.9) о разрешимом радикале асферических групп.

(4.1) Следующее утверждение является фактически переформулировкой полученных в $\oint 3$ результатов.

ПРЕДЛОЖЕНИЕ. Пусть $K$ - нетривиальная конечная подгруппа асферической про-р-группы $G$. Тогда ее нормализатор $N_{G}(K) \cong \mathbb{Z} / p^{m} \mathbb{Z}$.

ДоказАтЕльство. Так как по (2.6.(а)) группа $N_{G}(K)$ также асферична, то без ограничения общности можно считать, что $K \triangleleft G$.

Пусть $\bar{N}=\mathbb{F}_{p}\left(T, t^{0}\right)$ - пермутационный модуль соотношений группы $G$. По предложению (3.1) $K \subseteq G_{t}$ для некоторого $t \neq t^{0}$ из $T$. Тогда нормальность $K$ в группе $G$ дает, что $G_{t} \cap g G_{t} g^{-1} \supseteq K \neq\{1\}$ для произвольного $g \in G$. Так как $g G_{t} g^{-1}=G_{s}$, где $s=g \cdot t$, то вновь по $(3.1) g \cdot t=t$. Таким образом, $G=G_{t} \cong \mathbb{Z} / p^{m} \mathbb{Z}$. 
(4.2) СлЕДСтВИЕ. Абелева про-р-группа является асферической тогда и только тогда, когда она изоморфна одной из следующих групп: $\mathbb{Z} / p^{m} \mathbb{Z}, \mathbb{Z}_{p}$, $\mathbb{Z}_{p} \times \mathbb{Z}_{p}$

ДокАЗАтЕльство. Если в абелевой про-р-группе $G$ существует элемент $g \neq 1$ конечного порядка, то предыдущее предложение, примененное к подгрупше $K=\langle g\rangle$, дает $G \cong \mathbb{Z} / p^{m} \mathbb{Z}$.

Если $G$ без кручения, то, как хорошо известно, $G \cong \prod_{i \in I} \mathbb{Z}_{p}$. При этом $\operatorname{cd} G=|I|$ (см., например, [22]). Поэтому в силу следствия (3.6) из асферичности $G$ вытекает, что $|I| \leqslant 2$.

(4.3) ЛЕммА. Пусть $G$ - бесконечная асферическая про -р -группа, содержащая абелеву нормальную подгруппу $A \neq\{1\}$. Тогда в $G$ существует такая открытая нормальная подгруппа $G_{1}$ без кручения, содержсащая иентрализатор $C_{G}(A)$, что факторгруппа $G_{1} / C_{G}(A)$ не имеет кручения.

ДокАЗАтЕльство. В силу предложения (4.1) бесконечность $G$ влечет бесконечность $A$. Пусть $1 \neq g \in C_{G}(A)$ и $K=\langle g\rangle$. Тогда из $A \subseteq N_{G}(K)$ следует, что $|K|=\infty$. Таким образом, $C_{G}(A)$ - группа без кручения.

Факторгруппа $G / C_{G}(A)$ изоморфна подгруппе группы автоморфизмов Aut $A$. По следствию (4.2) группа $A$ изоморфна либо $\mathbb{Z}_{p}$, либо $\mathbb{Z}_{p} \times \mathbb{Z}_{p}$. Поэтому Aut $A$ изоморфна либо мультипликативной группе $\mathbb{Z}_{p}^{*}$ кольца целых $p$-адических чисел, либо группе $\mathrm{GL}\left(2, \mathbb{Z}_{p}\right)$. Так как обе эти группы почти без кручения, то в $G / C_{G}(A)$ также сушествует подгруппа $G_{1} / C_{G}(A)$ без кручения конечного индекса.

(4.4) ТЕОРемА. Пусть в асферической про-р-группе $G$ содержится нормальная подгруппа $A$, изоморфная $\mathbb{Z}_{p} \times \mathbb{Z}_{p}$. Тогда

(a) nодгруппа $A$ открыта в $G$ (в частности, $G$-разрешимая группа);

(b) иентрализатор $C_{G}(A)$ изоморфен $\mathbb{Z}_{p} \times \mathbb{Z}_{p}$, совпадает со своим иентрализатором в $G$ и является наибольшей нормальной абелевой подгруппой $G$.

ДокАЗАтЕльство. (1) Пусть $G_{1}$ - открытая подгруппа без кручения из леммы (4.3). Построим такую открытую подгруппу $H \subseteq G_{1}$, что $A \subseteq H$ и $H^{*} \cap A=A^{p}$.

Так как индекс $\left[A: A^{p}\right]$ конечен, то сушествует такая открытая нормальная подгруппа $U$ группы $G_{1}$, что $U \cap A \subseteq A^{p}$. Положим $H=\psi^{-1}(\psi(A))=A U$, где $\psi$ - канонический эпиморфизм $G_{1}$ на $G_{1} / U$.

(2) Докажем равенство $H=A$, из которого будет следовать открытость $A$ в групше $G$.

Предположим, что существует элемент $g \in H$, не принадлежащий $A$. Так как $H \subseteq G_{1}$, то $\langle g\rangle \cong \mathbb{Z}_{p}$. Если $\langle g\rangle \cap A=\{1\}$, то подгруппа $\langle g, A\rangle$ изоморфна $\left(\mathbb{Z}_{p} \times \mathbb{Z}_{p}\right) 入 \mathbb{Z}_{p}$ и имеет поэтому когомологическую размерность 3 (см. [22; гл. I, предложение 22]), что невозможно ввиду следствия (3.6). Поэтому образ $g$ в факторгруппе $G_{1} / A$ имеет конечный порядок $p^{m}, m \geqslant 1$. В силу того, что по лемме (4.3) $G_{1} / C_{G}(A)$ - группа без кручения, $g \in C_{G}(A)$.

Положим $h=g^{p^{m-1}}$. Тогда $h^{p} \in H^{*} \cap A=A^{p}$ и, следовательно, $h^{p}=d^{p}$ для некоторого $d \in A$. Так как $h \notin A$, то элемент $v=h^{-1} d$ из $C_{G}(A)$ нетривиален. С другой стороны, $v^{p}=h^{-p} d^{p}=1$. Получили противоречие с тем, что по лемме (4.3) группа $C_{G}(A)$ не имеет кручения. 
(3) Доказанная вьше конечность индекса $[G: A]$ означает, что $C_{G}(A)$ - группа, центр которой имеет конечный индекс. По известной теореме Шура это влечет конечность ее коммутанта $K$. В силу леммы $(4.3) K=\{1\}$. Таким образом, $C_{G}(A)-$ абелева асферическая про-р-группа, содержащая подгруппу, изоморфную $\mathbb{Z}_{p} \times \mathbb{Z}_{p}$. По следствию $(4.2) C_{G}(A) \cong \mathbb{Z}_{p} \times \mathbb{Z}_{p}$.

Теперь мы можем повторить все проведенные выше рассуждения для нормальной подгруппы $C_{G}(A)$ вместо $A$ и получить, что $C_{G} C_{G}(A) \cong \mathbb{Z}_{p} \times \mathbb{Z}_{p}$. Поэтому равенство $C_{G} C_{G}(A)=C_{G}(A)$ получится как следствие того факта, что $C_{G}(A)-$ наибольшая абелева нормальная подгруппа в $G$. Докажем это.

(4) Пусть $B$ - произвольная абелева нормальная подгруппа $G$. Докажем, что подгруппа $D=C_{G}(A) \cdot B$ абелева.

Предположим, что $B \cong \mathbb{Z}_{p} \times \mathbb{Z}_{p}$. Из открытости $A$ в $G$ следует, что $A_{0}=A \cap B$ изоморфна $\mathbb{Z}_{p} \times \mathbb{Z}_{p}$. Тогда, как показано выше, $C_{G}\left(A_{0}\right)$ - абелева группа и $D \subseteq$ $C_{G}\left(A_{0}\right)$.

Если $B=\langle b\rangle \cong \mathbb{Z}_{p}$, то из открытости $A$ в $G$ следует, что $b^{p^{m}} \in A$ для некоторого $m \geqslant 0$. Пусть $g \in C_{G}(A)$. Так как $B \triangleleft G$, то $g b g^{-1}=b^{\xi}$ для некоторого $\xi \in \mathbb{Z}_{p}^{*}$. Тогда $b^{p^{m}}=g b^{p^{m}} g^{-1}=\left(b^{p^{m}}\right)^{\xi}$ и, следовательно, $\xi=1$. Таким образом, абелевы подгруппы $B$ и $C_{G}(A)$ поэлементно коммутируют и, следовательно, $D$ абелева.

Теперь из абелевости $D$ и включения $A \subseteq C_{G}(A) \subseteq D$ следует, что $D \subseteq C_{G}(A)$. Таким образом, $C_{G}(A)$ содержит произвольную абелеву нормальную подгруппу $B$, что и требовалось доказать.

(4.5) ТЕОРемА. Пусть в асферической про-р-группе $G$ содерәсится нормальная подгруппа $A$, изоморфная $\mathbb{Z}_{p}$. Тогда

(а) существует содерәсащая $A$ подгруппа $G_{1}$ без кручения индекса $\left[G: G_{1}\right] \leqslant 2$

(b) в $G$ содержится нормальная подгруппа $H$ конечного индекса, являющаяся полупрямым произведением $H=A \lambda W$, где $W$ - свободная про -р -групnа;

(c) если $G$ без кручения, то

$$
d(G)=r(G)+1
$$

әде $d(G)$ и $r(G)$ - минимальные числа порожсдющих әруппь $G$ и соответственно ее определяющих соотношений.

Доказательство теоремы отложим до конца параграфа. Сейчас сделаем несколько замечаний о ее формулировке.

(1) При $p \neq 2$ утверждение (a) означает, что сама $G$ является группой без кручения.

(2) В утверждении (b) не исключается случай $W=\{1\}$, т.е. случай, когда $\operatorname{rank} W=0$. Кроме того произвольное полупрямое произведение $\mathbb{Z}_{p} \lambda W$ является асфферической про-р-группой (так как $\left.\operatorname{cd}\left(\mathbb{Z}_{p} \lambda W\right) \leqslant 2\right)$.

(3) Согласно [22] числа $d(G)$ и $r(G)$ равны размерностям $\operatorname{dim} H^{q}(G, \mathbb{Z} / p \mathbb{Z})$ над полем $\mathbb{F}_{p}$ при $q=1$ и $q=2$ соответственно. Поэтому равенство (1) в теореме следует понимать как равенство кардинальных чисел.

(4.6) Предыдущую теорему можно рассматривать как обобщение про- $p$-аналога теоремы Мурасуги о дискретных группах с одним определяюшим соотношением, содержащегося в утверждениях (a) и (c) следующей теоремы. 
ТЕОРЕма. Если $G$-неабелева асферическая про-р-группа с нетривиальным центром $Z(G)$, то

(a) $Z(G) \cong \mathbb{Z}_{p}$ и группа $G$ не имеет кручения;

(b) в $G$ содержсття нормальная подгруппа конечного индекса $H=$ $Z(G) \times W$, где $W-$ свободная про-р-группа $u \operatorname{rank} W \geqslant 1$;

(c) $d(G)=r(G)+1$.

ЗАмЕчАнИЕ. В утверждении (b) $\operatorname{rank} W=1$ только в одном случае: $p=2$ и $G$ - разрешимая про-2-группа с заданием

$$
G=\left\langle a, b \| b a b^{-1}=a^{-1}\right\rangle \cong \mathbb{Z}_{2} \lambda \mathbb{Z}_{2}
$$

При этом $Z(G)=\left\langle b^{2}\right\rangle$.

ДоказАТЕльство. Из леммы (4.3) следует, что $G$ - группа без кручения. Следствие (4.2) и теорема (4.4) при $A=Z(G)$ показывают, что из неабелевости группы $G$ вытекает изоморфизм $Z(G) \cong \mathbb{Z}_{p}$.

Теперь применение теоремы (4.5) дает утверждения (c) и (b) за исключением неравенства rank $W \geqslant 1$. Его справедливость вытекает из следующей ниже леммы (4.7), согласно которой $G \cong \mathbb{Z}_{p}$, если $W=\{1\}$.

Что касается замечания, то оно является следствием разрешимости $G$ при $W \cong \mathbb{Z}_{p}$ и доказываемого далее предложения (4.8.(b)).

(4.7) ЛЕмма. Пусть про-р-группа $G$ содерәсит открытую нормальную подгруппу $A \cong \mathbb{Z}_{p}$. Тогда для наибольшей конечной нормальной подгруппь $K$ группь $G$ либо $G / K \cong \mathbb{Z}_{p}$, либо $p=2$ и $G / K$ изоморфна бесконечной диэдральной про-2-гpynne

$$
\mathbb{D}\left(2^{\infty}\right)=(\mathbb{Z} / 2 \mathbb{Z}) *(\mathbb{Z} / 2 \mathbb{Z}) \cong \mathbb{Z}_{2} \lambda(\mathbb{Z} / 2 \mathbb{Z})
$$

ДокАЗАтЕльство. Сушествование $K$ вытекает из того, что порядки конечных подгрупп в $G$ ограничены сверху порядком $|G / A|<\infty$. Профакторизовав по $K$, будем в дальнейшем считать, что в $G$ не сушествует нетривиальных конечных нормальных подгрупп.

Предположим, что $G$ не изоморфна $\mathbb{Z}_{p}$ и что $A$ - максимальная среди изоморфных $\mathbb{Z}_{p}$ открытых нормальных подгрупп $G$. Из $[G: A]<\infty$ вытекает конечность индекса центра группы $C_{G}(A)$. Тогда по теореме Шура коммутант группы $C_{G}(A)$ является конечной нормальной в $G$ подгруппой и, следовательно, тривиален. Таким образом, $C_{G}(A)$ - конечно порожденная абелева про- $p$-группа. Поэтому ее периодическая часть конечна и инвариантна в $G$ и, следовательно, тривиальна. Так как в $C_{G}(A) \cong \prod_{i=1}^{n} \mathbb{Z}_{p}$ содержится открытая подгруппа $A \cong \mathbb{Z}_{p}$, то $C_{G}(A) \cong \mathbb{Z}_{p}$. Сделанное выше предположение о максимальности $A$ влечет равенство $C_{G}(A)=A$.

Сопряжение в $G$ дает изоморфизм $\lambda$ групшы $G / A$ на нетривиальную конечную $p$-подгруппу $\lambda(G / A)$ групшы $\mathbb{Z}_{p}^{*} \cong$ Aut $A$. Из строения мультипликативной группы $\mathbb{Z}_{p}^{*}$ кольца $\mathbb{Z}_{p}$ вытекает, что это возможно, лишш если $p=2$ и $\lambda(G / A)=\{ \pm 1\}$. Поэтому $G=\langle A, g\rangle$, где $g^{2}=b \in A$ и $g a g^{-1}=a^{-1}$ для любого $a \in A$. Тогда $b=g b g^{-1}=b^{-1}$, откуда $b=1$. Итак, $G=A \lambda\langle g\rangle \cong \mathbb{D}\left(2^{\infty}\right)$.

(4.8) Следуюшее утверждение послужит в дальнейшем базисом для получения полного описания класса разрешимых асферических про-р-групп (см. по этому поводу $§ 5)$. 
ПРЕДЛОЖЕНИЕ. Пусть $G$ - неабелева разрешимая асферическая про-ргpynna. Тогда

(а) либо $G$ содерэит открытую нормальную подгруппу, изоморфную $\mathbb{Z}_{p} \lambda \mathbb{Z}_{p}$, либо $p=2 u G \cong \mathbb{D}\left(2^{\infty}\right)$

(b) если $G$ без кручения, то $G \cong \mathbb{Z}_{p} \lambda \mathbb{Z}_{p}$.

ДокАЗАТЕльство. (а) В разрешимой группе $G$ содержится абелева нормальная подгруппа $A \neq 1$. В силу предложения (4.1) и следствия (4.2) $A$ изоморфна либо $\mathbb{Z}_{p} \times \mathbb{Z}_{p}$, либо $\mathbb{Z}_{p}$. В первом случае по теореме (4.4) сама подгруппа $A$ открыта в $G$. Во втором случае из теоремы (4.5) вытекает существование открытой подгруппы $H=A \lambda W$, где $W$ - свободная про-р-группа. В силу разрешимости $G$ $\operatorname{rank} W \leqslant 1$. Поэтому либо $H \cong \mathbb{Z}_{p} 入 \mathbb{Z}_{p}$, либо $W=\{1\}$ и подгруппа $A \cong \mathbb{Z}_{p}$ открыта в $G$. Применяем лемму (4.7) и получаем, учитывая предложение (4.1), что $p=2$ и $G \cong \mathbb{D}\left(2^{\infty}\right)$.

(b) Пусть $G$ - группа без кручения. Тогда из доказанного уже утверждения (a) вытекает сушествование в $G$ открытой подгруппы $H \cong \mathbb{Z}_{p} \lambda \mathbb{Z}_{p}$. Заметим, что $H-$ группа Демушкина ранга $d(H)=2$ (см. [23]), и воспользуемся следующим набором результатов обшего характера.

(i) Класс бесконечных групп Демушкина совпадает с классом групп Пуанкаре размерности 2 (см. [22]).

(ii) Класс групп Пуанкаре фиксированной размерности замкнут относительно перехода к конечным расширениям без кручения (см. [22; дополнение к гл. I]).

(iii) Эйлеровы характеристики группы $G$ и ее открытой подгруппы $H$ связаны соотношением $\chi(H)=\chi(G)[G: H]$ (см. [16]). При этом $\chi(G)=2-d(G)$, если $G-$ группа Демушкина.

В результате получаем, что $G$ - группа Демушкина ранга 2 . В силу [23] $G \cong$ $\mathbb{Z}_{p} \lambda \mathbb{Z}_{p}$

(4.9) ТеоремА. (а) Каждая асферическая про-р -группа $G$ обладает разреиимым радикалом $R(G)$.

(b) Если асферическая про-р -группа $G$ с нетривиальным радикалом $R(G)$ не разрешима, то $R(G) \cong \mathbb{Z}_{p}$ и группа $G$ обладает всеми свойствами из теоремь (4.5).

ДоказАТЕльство. (а) Если $|G|<\infty$, то $R(G)=G$. Пусть группа $G$ бесконечна и $N$ - нетривиальная разрешимая нормальная подгруппа в $G$. Применение леммы (4.3) к последнему нетривиальному члену $A$ ряда коммутантов $N$ дает сушествование в $G$ открытой нормальной подгрупшы $G_{1}$ без кручения. В силу предложения (4.1) $|N|=\infty$. Поэтому из предложения (4.8.(b)) следует, что $N_{1}=N \cap G_{1}$ изоморфна $\mathbb{Z}_{p} \lambda \mathbb{Z}_{p}$ или $\mathbb{Z}_{p}$. Таким образом, длины ряда коммутантов $\delta(N)$ разрешимых нормальных подгрупп $N$ группы $G$ ограничены сверху числом $2+\delta\left(G / G_{1}\right)$.

Последнее утверждение позволяет применить к множеству $\mathfrak{M}$ разрешимых нормальных подгрупп $G$ лемму Цорна и доказать существование в $\mathfrak{M}$ максимальных элементов. Пусть $M-$ один из них. Так как $N M \in \mathfrak{M}$ для любого $N \in \mathfrak{M}$, то $N \subseteq M$. Следовательно, $M=R(G)$ - наибольшая разрешимая нормальная подгруппа в $G$. 
(b) Предположим, что $R(G) \neq\{1\}$ и $R(G) \neq G$. Так же, как и в начале доказательства части (a), получаем сушествование в $R(G)$ открытой подгруппы $R_{1}$, которая нормальна в $G$ и изоморфна $\mathbb{Z}_{p} \lambda \mathbb{Z}_{p}$ или $\mathbb{Z}_{p}$.

Предположим, что $R_{1} \cong \mathbb{Z}_{p} \lambda \mathbb{Z}_{p}$. Если $R_{1} \cong \mathbb{Z}_{p} \times \mathbb{Z}_{p}$, то по теореме (4.4) $R_{1}$ открыта в $G$ и, следовательно, $G$ - разрешимая группа. Таким образом, группа $R_{1}$ не абелева. Тогда ее коммутант $A$ является, как легко видеть, изоморфной $\mathbb{Z}_{p}$ нормальной подгруппой в $G$. Отсюда в силу теоремы (4.5) вытекает сушествование в $G$ открытой подгруппы $H=A \lambda W$, где $W$ - свободная про-р-группа.

Через $\rho$ обозначим проекцию $H$ на $W$. Так как $H \cap R_{1}$ открыта в $R_{1}$, то $R_{1} \cap H \neq A=\operatorname{Ker} \rho$. Поэтому $\rho\left(R_{1} \cap H\right)$ является нетривиальной абелевой нормальной подгруппой свободной про- $p$-группы $W$. В частности, $\rho\left(R_{1} \cap H\right) \cong \mathbb{Z}_{p}$, откуда следует, что $W \cong \mathbb{Z}_{p}$ (см., например, [24; лемма 2.8]). Вопреки предположению группа $G$ оказывается разрешимой.

Таким образом, $R_{1} \cong \mathbb{Z}_{p}$. В силу леммы (4.7) для доказательства изоморфизма $R(G) \cong \mathbb{Z}_{p}$ достаточно только исключить случай $p=2, R(G) \cong \mathbb{D}\left(2^{\infty}\right)$.

Предположим, что радикал $R=R(G)$ равен $\langle a\rangle \lambda\langle b\rangle \cong \mathbb{D}\left(2^{\infty}\right)$. Так как $b^{2}=1$, то в силу предложения (4.1) получаем, что подгруппа $C_{G}(b)$ конечна и что из конечности содержашейся в ней нормальной в $G$ подгруппы $C_{G}(R)$ вытекает равенство $C_{G}(R)=\{1\}$.

В результате не разрешимая про-2-группа $G$ оказывается изоморфиой подгруппе группы автоморфизмов Aut $R$. Однако легко проверить, что 2-силовская подгруппа группы Aut $R$, где $R \cong \mathbb{D}\left(2^{\infty}\right)$, является разрешимой. В самом деле, коммутант $B=[R, R]$ равен $\left\langle a^{2}\right\rangle \cong \mathbb{Z}_{2}$ и открыт в $R$. Поэтому для стандартного гомоморфизма $\alpha$ групшы Aut $R$ в Aut $B \times \operatorname{Aut}(R / B)$ образ $\alpha(S)$ - разрешимая группа, а ядро $\operatorname{Ker} \alpha$, очевидно, абелево.

ДоКАЗАТЕЛЬСтво теоремы (4.5). (а) Согласно лемме (4.3) централизатор $C_{G}(A)$ не имеет кручения. Факторгруппа $G / C_{G}(A)$ изоморфна про-р-подгруппе группы Aut $A \cong \mathbb{Z}_{p}^{*}$. Как известно, $\mathbb{Z}_{p}^{*} \cong \mathbb{Z} /(p-1) \mathbb{Z} \times \mathbb{Z}_{p}$ при $p \neq 2$ и $\mathbb{Z}_{2}^{*} \cong \mathbb{Z} / 2 \mathbb{Z} \times \mathbb{Z}_{2}$. Поэтому либо $G / C_{G}(A)$ без кручения, либо $p=2$ и в $G / C_{G}(A)$ содержится подгруппа $G_{1} / C_{G}(A)$ без кручения индекса 2.

(b) (1) В силу утверждения (а) можно без ограничения общности считать, что $G$ - группа без кручения. Так же, как в начале доказательства теоремы (4.4), строим такую открытую нормальную в $G$ подгруппу $H$, что $A \subseteq H$ и $H^{*} \cap A=A^{p}$, где $H^{*}=H^{p}[H, H]$. Поэтому мы можем выбрать в $H$ минимальную систему порождающих $\left\{a, b_{y} \mid y \in Y\right\}$, где $\langle a\rangle=A$.

Пусть $F$ - свободная про-р-группа с базисом $X=\{z\} \cup Y$. Минимальное копредставление $\varphi: F \rightarrow H$ зададим равенствами $\varphi(z)=a, \varphi(y)=b_{y}$ для $y \in Y$. Положим $V=\langle Y\rangle, B=\varphi(V)$ и обозначим через $\psi$ ограничение $\left.\varphi\right|_{V}$. По построению $\alpha(B)=H / A$, где $\alpha$ - канонический эпиморфизм $H$ на $H / A$. Так как $V-$ свободная про-р-группа с базисом $Y$, то гомоморфизм $\tau=\alpha \psi$ группы $V$ на $H / A$ является копредставлением $H / A$. Обозначим $K=\operatorname{Ker} \tau$.

Нашей целью является доказательство того, что модуль соотношений $\bar{K}$ построенного копредставления является свободньгм $H / A$-модулем.

После этого можно будет, рассматривая последовательность модуля соотношений $\bar{K}$ как $\mathbb{F}_{p}(H / A)$-свободную резольвенту модуля $\mathbb{Z} / p \mathbb{Z}$, утверждать, что $\operatorname{cd}(H / A) \leqslant 2$. Тогда из предложения 22 [22; гл. I] вытекает равенство 
$1+\operatorname{cd}(H / A)=\operatorname{cd} H \leqslant 2$, откуда $\operatorname{cd}(H / A) \leqslant 1$. Таким образом, $H / A-$ свободная про-р-группа и, следовательно, $H=A \lambda W$, где $W \cong H / A$.

(2) Рассмотрим соответствующую копредставлению $\varphi: F \rightarrow H$ последовательность модуля соотношений (см. лемму (2.3))

$$
0 \longrightarrow \bar{N} \stackrel{\delta}{\longrightarrow} L_{X} \stackrel{\lambda}{\longrightarrow} \mathbb{F}_{p}(H) \stackrel{\varepsilon}{\longrightarrow} \mathbb{Z} / p \mathbb{Z} \longrightarrow 0
$$

Здесь $N=\operatorname{Ker} \varphi, L_{X}-$ свободный $H$-модуль с базисом $\left\{w_{x} \mid x \in X\right\}$. Так как $c d H \leqslant 2$ по следствию (3.6), то (2) является свободной $H$-резольвентой модуля $\mathbb{Z} / p \mathbb{Z}$.

В силу следствия (1.15) последовательность (2) можно рассматривать как свободную $A$-резольвенту $\mathbb{Z} / p \mathbb{Z}$. Тогда из $\operatorname{cd} A=1$ следует, что $\operatorname{Ker} \varepsilon-$ свободньй $A$-модуль. Поэтому $L_{X}=\operatorname{Im} \delta \oplus D$ для некоторого $A$-подмодуля $D$. СледовательHO,

$$
I(A) L_{X}=I(A) \operatorname{Im} \delta \oplus I(A) D
$$

откуда

$$
\operatorname{Im} \delta \cap I(A) L_{X}=I(A) \operatorname{Im} \delta .
$$

Обозначим $L_{X}^{\prime}=L_{X} / I(A) L_{X}$. Так как $A \triangleleft H$, то канонический эпиморфизм $\beta: L_{X} \rightarrow L_{X}^{\prime}$ является $H$-модульньм и из $(3)$ следует, что

$$
\beta(\operatorname{Im} \delta) \cong \bar{N} / I(A) \bar{N}
$$

Этот изоморфизм будем рассматривать как изоморфизм $H / A$-модулей. Из свободы $H$-модуля $\bar{N}$ вытекает свобода $\bar{N} / I(A) \bar{N}$ как $H / A$-модуля. Таким образом, $\beta(\operatorname{Im} \delta)$ - свободный $H / A$-подмодуль в $L_{X}^{\prime}$.

(3) Заметим, что $L_{X}^{\prime}$ является свободным $H / A$-модулем с базисом $\left\{w_{x}^{\prime} \mid x \in X\right\}$, где $w_{x}^{\prime}=\beta\left(w_{x}\right)$. Отображение $\beta$ в выбранных базисах явно задается следуюшим образом:

$$
\beta\left(\sum_{x \in X} \eta_{x} w_{x}\right)=\sum_{x \in X} \alpha\left(\eta_{x}\right) w_{x}^{\prime}
$$

Если $B \cap A=\{1\}$, то $H=A \lambda B$, откуда $\operatorname{cd}(H / A) \leqslant \operatorname{cd} H \leqslant 2$. Повторив рассуждения, приведенные в конце части (1) доказательства, получим, что $B$ свободная про- $p$-группа. Поэтому в дальнейшем будем считать, что $B \cap A=\langle d\rangle$, где $d=a^{p^{m}}$. Отметим, что здесь $m \geqslant 1$, так как $A$ не содержится в $B=\left\langle b_{y}\right|$ $y \in Y\rangle$.

Выберем теперь такой элемент $v \in V$, что $\psi(v)=d$. Естественная факторизация

$$
\varphi: F=\langle z\rangle * V \rightarrow A * B \rightarrow A \lambda B \rightarrow H
$$

гомоморфизма $\varphi$ показьвает, что $N=\operatorname{Ker} \varphi$ порождается как нормальная подгруппа в $F$ множеством

$$
R=\operatorname{Ker} \psi \cup\left\{v z^{-p^{m}}\right\} \cup\left\{y z y^{-1} s_{y}^{-1} \mid y \in Y\right\}
$$


где $s_{y}$ - элемент из $\langle z\rangle$, для которого $\varphi\left(s_{y}\right)=b_{y} a b_{y}^{-1}$. Поэтому из описания гомоморфизма $\delta$ в лемме $(2.3)$ следует, что подмодуль $\beta(\operatorname{Im} \delta)$ порождается в $L_{X}^{\prime}$ элементами

$$
\beta \delta(\bar{r})=\sum_{x \in X} \alpha \varphi\left(\frac{\partial r}{\partial x}\right) w_{x}^{\prime},
$$

где $r$ пробегает $R$.

(4) Представим $L_{X}=L_{z} \oplus L_{Y}$, где $L_{z}=\mathbb{F}_{p}(H) w_{z}, L_{Y}=\bigoplus_{y \in Y} \mathbb{F}_{p}(H) w_{y}$. Тогда $L_{X}^{\prime}=\beta\left(L_{z}\right) \oplus \beta\left(L_{Y}\right)$.

Если $r \in \operatorname{Ker} \psi \subseteq V$, то $\partial r / \partial z=0$ и $\partial r / \partial y \in \mathbb{F}_{p}(V)$. Следовательно, элементы

$$
\xi_{r}=\beta \delta(\bar{r})=\sum_{y \in Y} \tau\left(\frac{\partial r}{\partial y}\right) w_{y}^{\prime}
$$

принадлежат $\beta\left(L_{Y}\right)$.

Если $r=v z^{-p^{m}}$, то $\varphi(\partial r / \partial z)=-\varphi(v) a^{-p^{m}} \sigma$, где $\sigma=1+a+a^{2}+\cdots+a^{p^{m}-1}$. Из $m \geqslant 1$ вытекает, что $\sigma \in I(A) \subseteq \operatorname{Ker} \alpha$ и, следовательно, $\alpha \varphi(\partial r / \partial z)=0$. Так как $\partial r / \partial y=\partial v / \partial y \in \mathbb{F}_{p}(V)$, элемент

$$
\zeta=\beta \delta(\bar{r})=\sum_{y \in Y} \tau\left(\frac{\partial v}{\partial y}\right) w_{y}^{\prime}
$$

также принадлежит $\beta\left(L_{Y}\right)$.

Если $r=u z u^{-1} s_{u}^{-1}$ для некоторого $u \in Y$, то $\partial r / \partial y=0$ для всех $u \neq y \in Y$. Далее, $\varphi(\partial r / \partial u)=\varphi\left(1-u z u^{-1}\right)=1-b_{u} a b_{u}^{-1} \in I(A) \subseteq \operatorname{Ker} \alpha$. Таким образом, $\beta \delta(\bar{r}) \in \beta\left(L_{z}\right)$.

Из проведенных выше вычислений следует, что $\beta(\operatorname{Im} \delta)=M_{z} \oplus M_{Y}$, где $M_{z} \subseteq \beta\left(L_{z}\right), M_{Y} \subseteq \beta\left(L_{Y}\right)$. Поэтому из доказанной в части (2) свободы $H / A$-модуля $\beta(\operatorname{Im} \delta)$ вытекает свобода модуля $M_{Y}$.

(5) Теперь, вновь воспользовавшись леммой (2.3), построим последовательность модуля соотношений, соответствующую копредставлению $\tau: V \rightarrow H / A$. Участвующий в этой последовательности свободный $H / A$-модуль $L_{Y}^{\prime}$ с базисом $\left\{w_{y}^{\prime} \mid y \in Y\right\}$ можно отождествить с модулем $\beta\left(L_{Y}\right)$.

Из формул (4) и (5) следует, что при этом отождествлении подмодуль $M_{Y}$, порожденньй элементами $\zeta$ и $\xi_{r}, r \in \operatorname{Ker} \psi$, переходит в подмодуль $\operatorname{Im} \delta^{\prime}$ модуля $L_{X}^{\prime}$, которьй изоморфен модулю соотношений $\bar{K}$. В самом деле, нормальная подгруппа $K=\psi^{-1}(B \cap A)$ группы $V$ порождена подмножеством $\{v\} \cup \operatorname{Ker} \psi$.

Таким образом, $\bar{K} \cong M_{Y}-$ свободный $H / A$-модуль, что и требовалось доказать.

(c) Установим сначала справедливость равенства $d(H)=r(H)+1$ для открытой в $G$ подгруппы $H=A \lambda W$ из утверждения (b) теоремы.

Очевидно, что $d(H)=d(W)+1$. Далее, $H^{2}(H, \mathbb{Z} / p \mathbb{Z}) \cong H^{1}\left(W, H^{1}(A, \mathbb{Z} / p \mathbb{Z})\right) \cong$ $H^{1}(W, \mathbb{Z} / p \mathbb{Z})$ (см., например, [22; гл. I, п. 3.3]), откуда $r(H)=d(W)$.

Для переноса этого равенства на группу $G$ рассмотрим два случая.

(1) Числа $d(G)$ и $r(G)$ конечны. Так как по следствию (3.6) $c d G \leqslant 2$, то эйлерова характеристика $\chi(G)=1-d(G)+r(G)$. Как известно, $\chi(H)=[G: H] \chi(G)$. Поэтому из установленного выше равенства $\chi(H)=0$ получаем нужное нам $\chi(G)=0$. 
(2) Одно из чисел $d(G), r(G)$ бесконечно. В этом случае воспользуемся следующими неравенствами

$$
\begin{gathered}
r(G) \leqslant r(H) \leqslant[G: H] r(G), \\
d(G) \leqslant d(H)+d(G / H), \quad d(H) \leqslant[G: H] d(G)
\end{gathered}
$$

(первое неравенство в силу сd $G=2$ вытекает из сюръективности гомоморфизма коограничения cor: $\left.H^{2}(H, \mathbb{Z} / p \mathbb{Z}) \rightarrow H^{2}(G, \mathbb{Z} / p \mathbb{Z})\right)$.

Из двойного неравенства следует, что бесконечность одного из чисел $r(G), r(H)$ влечет равенство $r(G)=r(H)$. Такое же утверждение о числах $d(G)$ и $d(H)$ вытекает из остальных неравенств. Учитывая, что $d(H)=r(H)+1$, получаем отсюда, что в рассматриваемом случае имеет место равенство $d(G)=r(G)$, эквивалентное требуемому.

(4.10) ЗАмЕЧАнИЕ. Нетрудно проверить, что доказательства всех результатов этого параграфа используют только следующие внутренние свойства асферических про-р-групп $G$.

(C) Централизатор любого әлемента порядка р в группе $G$ является ииклической группой.

(D) Когомологическая размерность $\mathrm{cd} H \leqslant 2$ для любой подгруппь $H$ без кручения группь $G$.

Более того, из одного свойства $(\mathrm{C})$ про-р-группы $G$ без труда выводится вся информация о кручении в асферических группах, которая дается теоремой (3.1). А именно:

(a) любая конечная подгруппа $G$ является циклической;

(b) если $K_{1}$ и $K_{2}$ - конечные подгруппы $G$, то из $K_{1} \cap K_{2} \neq\{1\}$ следует, что либо $K_{1} \subseteq K_{2}$, либо $K_{2} \subseteq K_{1}$;

(c) если $K \cap g K g^{-1} \neq\{1\}$ для $g \in G$ и конечной подгрупшы $K$, то подгруппа $\langle K, g\rangle$ является циклической.

Поэтому было бы интересно исследовать возникший таким образом класс $(C D)$-групп и выяснить, насколько он шире класса асферических про-р-групп. В частности, можно поставить следующие вопросы.

1) Справедлива ли для $(C D)$-групп сформулированная во введении теорема В типа теоремы Линдона о тождествах или даже ее вариант для $\mathbb{Z}_{p}(G)$-модуля соотношений $N^{a b}=N /[N, N]$ ?

(Как отмечалось в пункте (3.3), из утверждения теоремы В не вытекает пермутационность модуля $\bar{N}$.)

2) Можно ли класс $(C D)$-групп охарактеризовать каким-либо свойством $\mathbb{Z}_{p}(G)$-модулей соотношений $N^{a b}$, близким к пермутационности?

\section{§5. О разрешимых асферических про- $p$-группах}

(5.1) Для краткости формулировок введем два рабочих термина. Будем называть про-p-группу стандартной, если она изоморфна одной из следующих групп:

(i) $\mathbb{Z}_{p}$ или $\mathbb{Z} / p^{m} \mathbb{Z}, m \geqslant 0$;

(ii) групше Демушкина $\mathbb{Z}_{p} 入 \mathbb{Z}_{p}$ ранга 2 ;

(iii) в случае $p=2$ бесконечной диэдральной про-2-группе $\mathbb{D}\left(2^{\infty}\right)$. 
Асферичность стандартных про-р-групп вытекает из теорем (2.6), (2.7) и следствия (2.9).

Для малых $p$ зададим порождающими и определяющими соотношениями четыре про- $p$-группы, которые назовем здесь специальными. А именно, про-3-групша

$$
E_{3}(3)=\left\langle x_{1}, x_{2}, x_{3} \| x_{1}^{3}=x_{2}^{3}=x_{3}^{3}=x_{1} x_{2} x_{3}=1\right\rangle
$$

и про-2-групшы

$$
\begin{aligned}
& E_{2}(2)=\left\langle x_{1}, x_{2}, y \| x_{1}^{2}=x_{2}^{2}=x_{1} x_{2} y^{2}=1\right\rangle \\
& E_{2}(3)=\left\langle x_{1}, x_{2}, x_{3} \| x_{1}^{2}=x_{2}^{4}=x_{3}^{4}=x_{1} x_{2} x_{3}=1\right\rangle \\
& E_{2}(4)=\left\langle x_{1}, x_{2}, x_{3}, x_{4} \| x_{1}^{2}=x_{2}^{2}=x_{3}^{2}=x_{4}^{2}=x_{1} x_{2} x_{3} x_{4}=1\right\rangle
\end{aligned}
$$

Следствие (2.9) позволяет установить асферичность групп $E_{2}(j), j=2,4$. Строение специальных групп и, в частности, их разрешимость видны из следуюшего их описания:

$$
E_{3}(3)=\langle a, b\rangle \lambda\langle c\rangle \cong\left(\mathbb{Z}_{3} \times \mathbb{Z}_{3}\right) \lambda(\mathbb{Z} / 3 \mathbb{Z}), \quad c a c^{-1}=b, \quad c b c^{-1}=a^{-1} b^{-1}
$$

(это представление получается из исходного с помощью преобразований Титце после замены порождающих по формулам $x_{1}=c a, x_{2}=c, x_{3}=c a^{-1} c$ );

$$
\begin{gathered}
E_{2}(2)=\langle a, b\rangle \lambda\langle c\rangle \cong\left(\mathbb{Z}_{2} \lambda \mathbb{Z}_{2}\right) \lambda(\mathbb{Z} / 2 \mathbb{Z}) \\
b a b^{-1}=a^{-1}, \quad c a c^{-1}=a^{-1}, \quad c b c^{-1}=b^{-1} a
\end{gathered}
$$

(здесь $\left.x_{1}=b^{-2} c, x_{2}=c, y=b\right)$;

$$
E_{2}(3)=\langle a, b\rangle \lambda\langle c\rangle \cong\left(\mathbb{Z}_{2} \times \mathbb{Z}_{2}\right) \lambda(\mathbb{Z} / 4 \mathbb{Z}), \quad c a c^{-1}=b, c a c^{-1}=a^{-1}
$$

(здесь $\left.x_{1}=a c^{2}, x_{2}=c, x_{3}=c a^{-1}\right)$;

$$
E_{2}(4)=\langle a, b\rangle \lambda\langle c\rangle \cong\left(\mathbb{Z}_{2} \times \mathbb{Z}_{2}\right) \lambda(\mathbb{Z} / 2 \mathbb{Z}), \quad c a c^{-1}=a^{-1}, \quad c b c^{-1}=b^{-1}
$$

(здесь $\left.x_{1}=a c, x_{2}=c b, x_{3}=c, x_{4}=(a b c)^{-1}\right)$.

(5.2) Анонсируем справедливость следуюшего описания класса разрешимых асфферических про-р-групп.

ТЕОРема. Разрешимая про-р-группа является асферической тогда и только тогда, когда она либо стандартная, либо специальная группа.

Эта теорема представляет собой следствие решения намного более общей классификационной задачи, которое будет получено в следуюшей публикации.

Однако “бо́льшая часть" теоремы может быть доказана с помощью самых элементарных фактов линейной алгебры. Ниже приводится это простое рассуждение.

(5.3) Сформулированная во введении теорема D вытекает из следуюшего предложения и сделанного выше замечания об асферичности стандартных групп. 
ПРЕДЛОЖЕНИЕ. Пусть $p \neq 2$. Если $G$ - разрешимая асферическая про-р группа, не являющаяся стандартной, то $p=3$ и $G \cong E_{3}(3)$.

ДоказАтельство. (1) Так как $G$ не является стандартной, то из следствия (4.2) и предложения (4.8) вытекает, что в $G$ имеются элементы порядка $p$ и существует открытая нормальная подгруппа $H \cong \mathbb{Z}_{p} \lambda \mathbb{Z}_{p}$. Если бы $H$ была неабелева, то ее коммутант был бы нормальной подгруппой в $G$, изоморфной $\mathbb{Z}_{p}$. Тогда из теоремы (4.5.(а)) следовало бы, что $G$ - группа без кручения.

Таким образом, $H \cong \mathbb{Z}_{p} \times \mathbb{Z}_{p}$. Применяя теорему (4.4), получаем нормальную подгруппу $W=C_{G}(H)$, изоморфную $\mathbb{Z}_{p} \times \mathbb{Z}_{p}$, обладающую свойством $C_{G}(W)=W$. Сопряжение в $G$ определяет изоморфизм $G / W$ на нетривиальную конечную $p$-подгруппу $K$ группы Aut $W \cong \mathrm{GL}\left(2, \mathbb{Z}_{p}\right)$. Следующее далее описание таких подгрупп без сомнения хорошо известно.

(2) Сначала воспользуемся следующими фактами:

(i) при $p \neq 2$ ядро стандартного эпиморфизма $\operatorname{GL}\left(2, \mathbb{Z}_{p}\right)$ на $\operatorname{GL}\left(2, \mathbb{F}_{p}\right)$ не имеет кручения;

(ii) группа унитреугольных матриц $U T\left(2, \mathbb{F}_{p}\right)$ имеет порядок $p$ и является $p$-силовской подгруппой в $\mathrm{GL}\left(2, \mathbb{F}_{p}\right)$.

Отсюда, прежде всего, вытекает, что $K \cong \mathbb{Z} / p \mathbb{Z}$ и, следовательно, $G=W \lambda\langle c\rangle$, где $c$ - произвольно выбранный в $G$ элемент порядка $p$. Далее, в векторном $\mathbb{F}_{p}$-пространстве $\bar{W}=W / W^{*}$ существует базис $\{\bar{v}, \bar{w}\}$, в котором сопряжению с помощью элемента $c$ соответствует матрица $\bar{C} \in U T\left(2, \mathbb{F}_{p}\right)$. Иначе, $\bar{C} \bar{v}=\bar{v}, \bar{C} \bar{w}=\gamma \bar{v}+\bar{w}$ для некоторого $0 \neq \gamma \in \mathbb{F}_{p}$. Ясно, что $\{\bar{w}, \bar{C} \bar{w}\}$ также является базисом $\bar{W}$. Поэтому если $a$ - произвольный прообраз в $W$ элемента $\bar{w} \in W / W^{*}$, то $W=\langle a, b\rangle$, где $b=c a c^{-1}$.

(3) Будем считать, что $W$ вложено в векторное пространство $W \otimes \mathbb{Q}_{p}$ над полем $p$-адических чисел с базисом $\{a, b\}$. Пусть действию элемента $c \in G$ на $W$ соответствует матрица $A \in \mathrm{GL}\left(2, \mathbb{Z}_{p}\right)$. Через $f_{A}(t)$ обозначим минимальньй многочлен $A$ над $\mathbb{Q}_{p}$, т.е. порождающий элемент идеала

$$
I_{A}=\left\{h(t) \in \mathbb{Q}_{p}[t] \mid h(A)=0\right\} .
$$

Заметим теперь, что

(iii) характеристический многочлен $P_{A}(t)=\operatorname{det}(t E-A)$ принадлежит $I_{A}$ и имеет степень 2 ;

(iv) так как $|c|=p$, то многочлен $t^{p}-1=(t-1) g(t)$ принадлежит $I_{A}$;

$(\mathrm{v})$ многочлен $g(t)=t^{p-1}+t^{p-2}+\cdots+1$ неприводим ( так как многочлен $g(x+1)$ из $\mathbb{Q}_{p}[x]$ удовлетворяет критерию Эйзенштейна).

Так как векторы $a$ и $A a=b$ не пропорциональны, степень $\operatorname{deg} f_{A}(t) \geqslant 2$. Поэтому в силу (iii) $\operatorname{deg} f_{A}(t)=2$. Так как $p \geqslant 3$, то $f_{A}(t) \neq t^{p}-1$ и в силу (iv) и (v) $f_{A}(t)=g(t)$.

Отсюда, прежде всего, следует, что $p=3$. Далее, равенство $A^{2}+A+E=$ $f_{A}(A)=0$ означает, что $c^{2} a c^{-2}=a^{-1}\left(c a c^{-1}\right)^{-1}$ или, иначе, $c b c^{-1}=a^{-1} b^{-1}$. Таким образом, $G \cong E_{3}(3)$. 
ЗАмечАниЕ. Подобное доказательство в случае $p=2$ должно включать исследование элементов порядка 2 в группах автоморфизмов неабелевых групп $\mathbb{Z}_{2} 入 \mathbb{Z}_{2}$ и быть более разветвленным в части, касающейся 2 -подгрупп из $\mathrm{GL}\left(2, \mathbb{Z}_{2}\right)$.

Результаты, которые планируется поместить в упоминавшееся вьше продолжение статьи, анонсированы в [25].

\section{Список литературы}

1. Линдон P., Шупn П. Комбинаторная теория групп. М.: Мир, 1980.

2. Huebschmann J. Cohomology theory of aspherical groups and of small cancellation groups // J. Pure Appl. Algebra. 1979. V. 14. № 2. P. 137-143.

3. Chiswell I. M., Collins D. J., Huebschmann J. Aspherical group presentations // Math. Z. 1981. V. 178. № 1. P. 1-36.

4. Кох X. Теория Галуа $p$-расширений. М.: Мир, 1973.

5. Мельников $О$. В. Подгруппы и гомологиии свободных произведений проконечных групा // Изв. АН СССР. Сер. матем. 1989. Т. 53. №1. С. 97-120.

6. Gildenhuys D., Ribes L. Profinite groups and Boolean graphs // J. Pure Appl. Algebra. 1978. V. 12. № 1. P. 21-47.

7. Залесский П.А., Мельников О.В. Подгрупшы проконечных групा, действующих на деревьях // Матем. сб. 1988. Т. 135. №4. С. 419-439.

8. Залесский П.А., Мельников О.В. Фундаментальные группы граффов проконечных групп // Алгебра и анализ. 1989. Т. 1. №4. С. 117-135.

9. Brumer A. Pseudo-compact algebras, profinite groups and class formations // J. Algebra. 1966. V. 4. № 3. P. 442-470.

10. Мельников О.В. Факторгруппы проконечных групп с двойственностью Пуанкаре // Изв. АН Беларуси. Сер. физ.-матем. наук. 1996. № 3. С. 54-58.

11. Gruenberg K. W. Projective profinite groups // J. London Math. Soc. 1967. V. 42. № 1 . P. $155-165$.

12. Gildenhuys D., Lim C.-K. Free pro-C C-groups // Math. Z. 1972. V. 125. № 1. P. 233-254.

13. Fox R. H. Free differential calculus. I. Derivation in the free group ring // Ann. of Math. (2). 1953. V. 57. № 3. P. 547-560.

14. Gruenberg K.W. Cohomological topics in group theory // Lecture Notes in Math. V. 143. Berlin: Springer-Verlag, 1970.

15. Басс X. Алгебраическая $K$-теория. М.: Мир, 1973.

16. Serre J.-P. Cohomologie des groupes discrets // Ann. of Math. Stud. 1971. V. 70. P. $77-169$.

17. Бурбаки Н. Алгебра. Гл. Х. Гомологическая алгебра. М.: Наука, 1987.

18. Ribes L. On amalgamated products of profinite groups // Math. Z. 1971. V. 123. № 4 . P. 357-374.

19. Binz E. N., Neukirch J., Wenzel G.H. A subgroup theorem for free products of pro-finite groups // J. Algebra. 1971. V. 19. № 1. P. 104-109.

20. Haran D. On closed subgroups of free products of profinite groups // Proc. London Math. Soc. 1987. V. 123. № 2. P. 266-298.

21. Мельников О.В. О свободных произведениях абсолютных групп Галуа // Сиб. матем. журн. 1999. Т. 40. №1. С. 113-118.

22. Серр Ж.-П. Когомологии Галуа. М.: Мир, 1968.

23. Labute J. P. Classification of Demushkin groups // Canad. J. Math. 1967. V. 19. № 1. P. 106-132.

24. Мельников О.В. Нормальные делители свободных проконечных групп // Изв. АН СССР. Сер. матем. 1978. Т. 42. № 1. С. 3-25.

25. Мельников О.В., Шишкевич A.A. Про-р-групшы с виртуальной двойственностью Пуанкаре размерности 2 // Докл. НАН Беларуси. 2002. Т. 46. № 1. С. 13-15.

Белорусский государственный университет, г. Минск 